\title{
Ranking Tourist Attractions through Online Reviews: A Novel Method with Intuitionistic and Hesitant Fuzzy Information Based on Sentiment Analysis
}

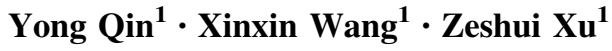

Received: 24 June 2020 / Revised: 28 May 2021/Accepted: 31 May 2021 / Published online: 24 June 2021

(C) Taiwan Fuzzy Systems Association 2021

\begin{abstract}
Online tourist reviews are the real feeling of tourists after the journey, which have a strong reference value for potential tourists to make travel decisions. However, it is almost impossible for a potential tourist to look through the massive online reviews related to tourist attractions (TAs) so that he/she can make the most appropriate decision. To this end, this paper proposes a recommender system to rank the alternative TAs through online reviews based on aspect-level sentiment analysis and multi-criteria decision-making (MCDM) with intuitionistic and hesitant fuzzy information. In this methodology, the aspects that the experienced tourists concern are extracted from online reviews to construct a three-level evaluation system (including target layer, criteria layer and sub-criteria layer), which not only ensures the comprehensive evaluation of TAs as much as possible, but also reduces the complexity of the decision-making process. Then, the online reviews related to these sub-criteria are transformed into the corresponding intuitionistic and hesitant fuzzy performance scores through aspect-level sentiment analysis. Furthermore, in order to obtain the final ranking result that more in line with the expectations of the potential tourist, the preference information from the potential tourist and experienced tourists is integrated to determine the weights of criteria. Subsequently, the
\end{abstract}

\section{Zeshui Xu}

xuzeshui@263.net

Yong Qin

yongqin_ahsc@163.com

Xinxin Wang

wangxinxin_cd@163.com

1 Business School, Sichuan University, Chengdu 610064, China intuitionistic and hesitant fuzzy TOPSIS (IHF-TOPSIS) method is proposed to rank the alternative TAs. Finally, a case study is provided to verify the validity and applicability of the methodology.

Keywords Tourist attractions - Massive online reviews . Aspect-level sentiment analysis · Preference information . IHF-TOPSIS method

\section{Introduction}

With the accelerating informatization process, social media and the Internet have been applied to disseminate various types of tourism-related information in recent years. Especially by efficient and real-time motivation, a large number of online reviews on TAs have been produced, which has gradually benefited tourists in expressing their appeals and reflecting their perceptions of TAs $[1,2]$. For example, online travel websites (OTWs) such as TripAdvisor (https://www.tripadvisor.cn), Qunar (https://www. qunar.com) and Ctrip (https://www.ctrip.com) have set up many channels to encourage tourists to express their feelings and experience during the journey. These massive online reviews appear on different OTWs in various forms and have become an increasingly important carrier of experience information. Related studies have shown that online reviews from experienced tourists have a profound impact on the choice of destinations for potential tourists $[3,4]$. What's more, the tourists often encounter so many alternative TAs with less cost or better experience quality. As a result, more and more tourists choose to collect online reviews of TAs before traveling and assist their travel decisions according to their own preferences. However, with the vigorous development of the global tourism 
industry, the number of tourists has increased stepwise, which has led to a rapid increase in the number of online reviews. It is difficult and even impossible for potential tourists to browse and analyze each review in detail. At the same time, the reviews from experienced tourists tend to be highly arbitrary, and the forms are diverse. What is worse, a large amount of redundant and meaningless information interferes with the choices and judgments of potential tourists, which has caused serious information overload problems. As a result, it is challenging for potential tourists to make the most appropriate personalized travel decisions directly [5]. Therefore, in order to solve the travel decisionmaking problem of potential tourists, it is necessary to develop a decision-making aid for ranking TAs through online reviews. Using the method in this paper, the potential tourists can identify the sentiment orientation of each online review, and analyze the performance of each TA in various aspects, and finally determine the comprehensive ranking of each TA. In this way, the potential tourists can make the best travel decisions based on their own preferences and the actual operation status of each TA.

Online tourist reviews often involve information on products, services, environment and other aspects about TAs, and reflect strong personal sentiment orientations as well. Therefore, using efficient and reliable methods will greatly simplify the operation and reduce the difficulty of decision-making for potential tourists to extract the sentiment orientations related to these aspects. However, the number of online reviews produced by tourists is too large, meanwhile, the sentence structure is complex and lacks organization, which makes it difficult to solve such problems manually. In recent years, with the rise of the natural language processing (NLP) technique, the sentiment orientation analysis technique has emerged to solve the above problems and has grown into one of the most active research fields in NLP [6].

The ranking of alternatives is usually considered as a multi-criteria decision-making (MCDM) problem. Up to now, some scholars have made prominent contributions to the problem of ranking alternatives through online reviews based on sentiment analysis. They realized that the information in online reviews is not always possible to express it with numerical ratings due to their ambiguity and uncertainty [7]. Therefore, fuzzy sets theory has been popular to deal with this problem. Among them, the intuitionistic fuzzy sets (IFS) theory has been widely accepted by many scholars [8]. The online reviews related to each criterion are divided into three categories (i.e., positive, neutral and negative) based on the sentiment analysis technique, which exactly corresponds to membership, hesitation and non-membership information in an IFS. As a result, the performance score of each criterion can be characterized by an intuitionistic fuzzy number (IFN). However, it cannot characterize the hesitation information of online reviewers. For this, the hesitant fuzzy sets (HFS) theory can be utilized to solve this problem well because it allows different memberships for a single criterion [9]. Meanwhile, most of the existing methods for ranking alternatives through online reviews contain only several evaluation criteria, usually given by experts or potential consumers, which may result in an incomprehensive evaluation. In this paper, the aspects that the experienced tourists concern are extracted from massive online reviews to construct a three-level evaluation system, including target layer, criteria layer and sub-criteria layer. Furthermore, few existing studies both consider the preference information from the potential consumer and online reviewers, which easily leads to a deviation in the final ranking. Therefore, the weights of criteria are determined together by both the potential consumer and online reviewers in this study.

The goal of this paper is to develop a recommender system (RS) to rank TAs through online reviews based on aspect-level sentiment analysis and MCDM with intuitionistic and hesitant fuzzy information. The potential tourists can utilize the RS to select the most suitable TA efficiently according to their own preferences, and it can be divided into four parts. Firstly, online reviews of alternative TAs from OTWs are obtained by using web crawler software. Secondly, the aspects that the experienced tourists concern from online reviews are extracted to construct a three-level evaluation system, and the aspect-level sentiment analysis is applied to identify the intuitionistic and hesitant fuzzy information contained in online reviews. As a result, a HF-decision matrix is built. Third, the weights of criteria are determined by the preference information from the potential tourist and experienced tourists. Finally, an intuitionistic and hesitant fuzzy TOPSIS (IHF-TOPSIS) method is proposed to rank alternative TAs. Therefore, the main contributions of this work to the existing body of knowledge can be concluded as follows:

(1) Both the ambiguity and hesitancy in online tourist reviews are effectively extracted and characterized in this paper building on the aspect-level sentiment technique, IFS theory and HFS theory. More precisely, this study provides a practical idea for converting massive qualitative review information into intuitionistic and hesitant fuzzy quantitative information, thus facilitating the subsequent flexible operations for decision making.

(2) To ensure the reliability of the final ranking and avoid the flaws of subjective or objective single weighting, this paper fully considers the preference information of potential tourist and experienced 
tourists for each criterion, and integrates them based on the principle of deviation minimization to form more accurate weighting information.

(3) According to the above techniques and tactics, this paper further proposes a novel MCDM procedure, namely IHF-TOPSIS. It requires that the initial decision information comes from a large number of experienced tourists. For one thing, the wisdom of massive experienced tourists is pooled to minimize decision bias. For another, the traditional decision matrix is usually evaluated by several experts, which is simple to operate but highly subjective at the same time. The initial evaluation information in this paper is from the real reviews associated with tourists, thus it can make up for this drawback well.

The rest of this paper is structured as follows: In Section "Related works", an overview of the related works is provided. In Sect. 3, the problem of ranking TAs through online reviews is described. In Sect. 4, the process of the IHF-TOPSIS method based on aspect-level sentiment analysis is interpreted step by step. In Sect. 5, a case study is given to verify the validity of the RS. In Sect. 6, conclusions and some discussions are summarized.

\section{Related Works}

Based on the aspect-level sentiment analysis, fuzzy sets and fuzzy preference relations, this paper aims to rank alternative TAs through online reviews. Therefore, the related works are divided into three aspects, i.e., (1) the research on sentiment analysis; (2) the research on fuzzy sets and fuzzy preference relations; (3) the research on ranking alternatives through online reviews. A brief literature review of each aspect is presented below.

\subsection{Sentiment Analysis}

Sentiment analysis, also known as opinion mining, orientation analysis, etc., has been a new analytical method in NLP in recent years. It is mainly used for analyzing the texts with emotional color and to judge the sentiment polarity. Therefore, it can offer an insight to support decision-makers [10].

Sentiment polarity judgment (i.e., sentiment classification) can generally be divided into binary classification (positive and negative), ternary classification (positive, negative and neutral) or multi-class sentiment classification problems [11]. Thus, how to improve the accuracy of classification results has become the focus of many scholars [12, 13]. Over the past few years, a number of techniques and methods for sentiment analysis have been proposed [14], such as the naive Bayes method [15], the support vector machine (SVM) algorithm [16] and the deep-learning technique [17]. According to the existing research, the main sentiment classification methods are divided into four categories (See Fig. 1): lexicon-based, machine learning, weak labeling-based and deep learning [18, 19].

With the development of the deep learning technique in recent years, the sentiment classification method based on deep learning has gradually attracted people's attention. Compared with the traditional sentiment classification method, it has a high generalization ability, which can automatically learn the deep semantic and syntactic features, and still maintain a high effect on relatively long sentences without requirements for high-level feature engineering, so that the overall classification results are more accurate [20]. At present, there are some well-known artificial intelligence (AI) service platforms in the world, such as Baidu, Google, Tencent, etc. It not only provides a large number of AI services, including opinion extraction, sentiment analysis, text similarity analysis, etc., but also is freely available to the public. Among them, the Baidu AI platform has been applied in a wide range of fields, and the accuracy rate of sentiment classification based on deep learning is as high as $95 \%$ or more. Therefore, the sentiment classification based on the Baidu AI platform is carried out in this paper to ensure the effectiveness of the classification results.

According to the requirements of different problems, sentiment analysis is divided into three levels: documentlevel, sentence-level and aspect-level. The document level identifies the sentiment polarity of the whole document, and it is considered as the most basic task. The sentencelevel identifies the sentiment polarity in each sentence of text, which is regarded as a subtask of document-level. Both of the above two levels of sentiment analysis are devoted to the polarity classification of opinions, but ignore the extraction of evaluation objects or targets [10]. This problem is well solved by the proposal of aspect-level sentiment analysis (i.e., fine-grained level). It can not only extract all aspects (i.e., targets) in a sentence, but also identify the sentiment polarity corresponding to each aspect. For example, in a review, the tourists may express their opinions on the transportation, service, food and other aspects of the TA where they have already experienced. These aspects extracted from the reviews may be classified into positive, neutral, negative or other more categories. In order to get more evaluation information in online reviews, aspect-level sentiment analysis is utilized in this paper. 


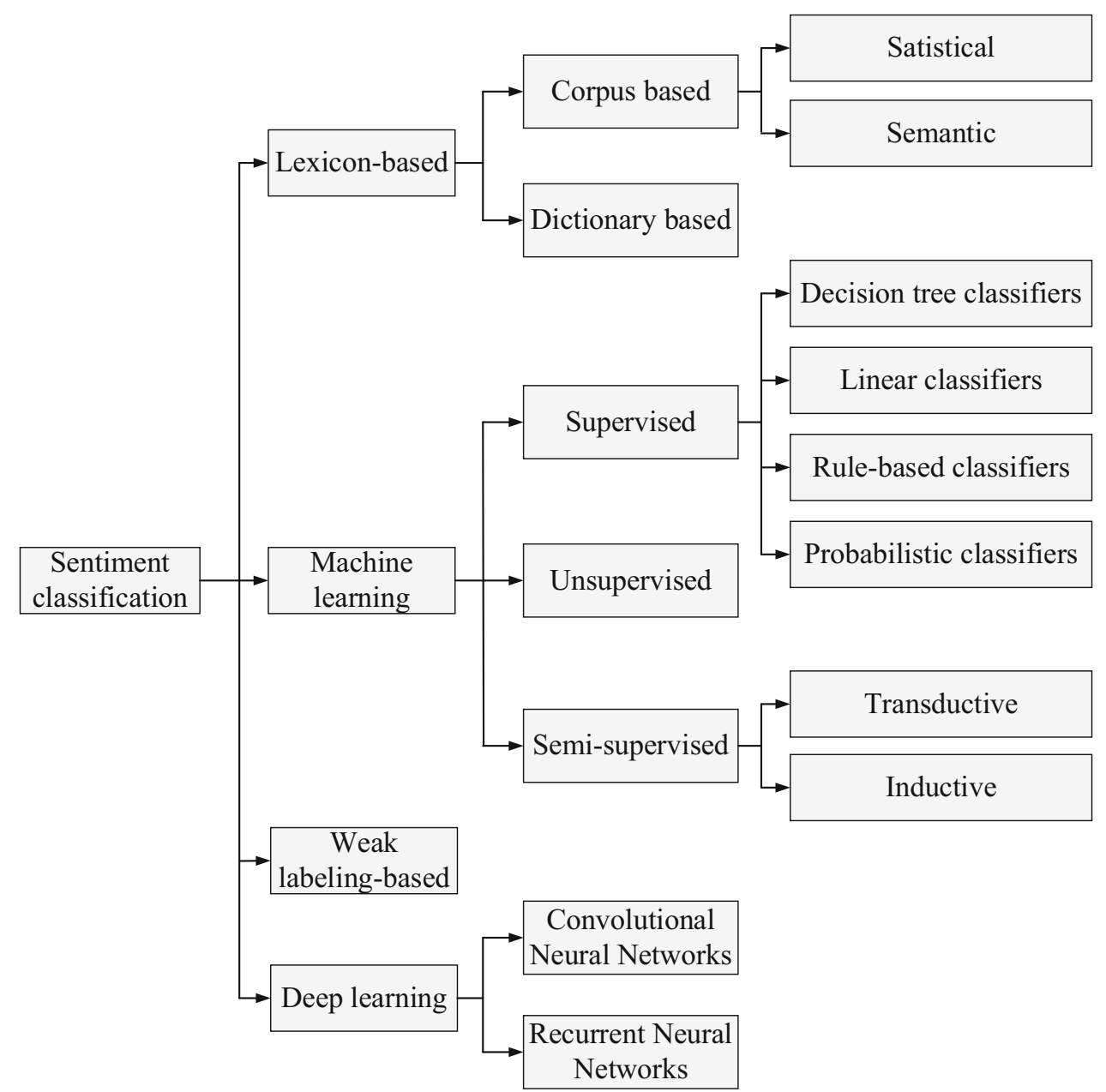

Fig. 1 The methods of sentiment classification

\subsection{Fuzzy Sets and Fuzzy Preference Relations}

Due to the limitation of human cognition, many problems in real life are often difficult to describe with precise numbers. To this end, Zadeh [21] proposed the fuzzy sets theory to characterize the uncertainty in decision-making. From the perspective of membership degree, fuzzy sets fully consider that the membership degree of the decision objects to an attribute or criterion. After that, many scholars extended this theory and proposed some new forms of fuzzy sets, such as interval fuzzy sets [22], IFS [23], HFS [24], Pythagorean fuzzy sets [25], etc. Among them, IFS theory and HFS theory are more in line with people's cognitions, so they have received extensive attention from scholars.

IFS, introduced by Atanassov [23], which contains information in three dimensions: membership degree, nonmembership degree and hesitation degree. As a result, it can more vividly describe the perception of decisionmakers on decision objects and provide the possibility of measuring uncertainty and hesitation [26, 27]. The past few decades have seen the rapid development and applications of IFS theory in many facets. Building on IFS, many MCDM methods have been proposed one after another, such as a novel EDAS approach for assessment of healthcare waste disposal technology [28], a new approach to multi-criteria decision-making [29] and the ELECTRE multi-criteria analysis approach for ranking alternatives [30]. Alternatively, IFS has found an increasingly wide utilization in a wide range of fields, such as image fusion [31], pattern recognition [32] and energy evaluation [33]. Moreover, considering that a single membership degree may not express the decision information well in some situations. Hence, HFS theory was proposed and developed, several possible membership degrees are included, to depict the hesitation and preference of decision-makers $[24,34,35]$. In contrast to the traditional fuzzy set, HFS presents a lot of superiorities to deal with uncertainty. Thus, the issue of this topic has received considerable attention. Xia and $\mathrm{Xu}$ [34] introduced the mathematical expression of HFS as well as the notion of hesitant fuzzy element (HFE). At the same time, a series of hesitant fuzzy 
information aggregation operators and their application in decision-making were also discussed. Afterwards, several hesitant fuzzy hybrid weighted aggregation operators were ulteriorly put forth by Liao and $\mathrm{Xu}$ [36]. In addition, the diverse distance measures for HFS were proposed, based on which, the corresponding similarity measures also were defined [37]. Later, Xu and Xia [38] put forward the correlation measures for hesitant fuzzy information and gave an intensive study on their properties. Similarly, a lot of MCDM methods based on hesitant fuzzy information have been developed and formed to handle many practical problems. For instance, a novel hesitant fuzzy WASPAS method was developed to solve the green supplier selection problem [39]. To evaluate the service quality of airline, Liao and $\mathrm{Xu}$ [40] introduced the hesitant fuzzy VIKOR method. What is more, the hesitant fuzzy-SWARA-WASPAS approach was conducted to appraise the key challenges of digital health interventions adoption during the COVID-19 outbreak [41].

In the process of making decisions, decision-makers need to evaluate and analyze the objectives, and individual preferences will be reflected in the evaluation process. In general, there are three methods used to describe the preferences of decision-makers: ordering preference [42], utility value [43] and preference relations [44]. Ordering preference depicts preference using the natural numbers to rank the alternatives from high to low according to the preferences of decision-makers. Utility value depicts preference by using a column of numbers between 0 and 1 to represent the preference degree of the decision-maker for different alternatives. Preference relations depict preference by constructing a comparison matrix to reflect that the degree of one alternative is superior to the other (i.e., the preference degree). In the three methods described above, the amount of information provided by ordering preference is too small, and utility value cannot be effectively measured. However, preference relations can solve the above problems well. It is not only simple to operate, but also can clearly express the preference information in decision-making according to the cognition of decisionmakers. Therefore, it has been widely adopted by many scholars.

Preference relations are also applicable to the fuzzy environment. Considering that decision-makers have limited knowledge of decision objectives in the actual decision-making, it is difficult to give accurate preference values. Therefore, this paper utilizes the intuitionistic fuzzy preference relations (IFPR) to characterize the preference information of potential tourists, and expresses the preference of potential tourists for each criterion from three aspects: "better", "unsure" and "not better" [45].

\subsection{Studies on Ranking Alternatives Through Online Reviews}

Although sentiment analysis techniques have been proposed and tended to mature, the issue of ranking alternatives by combining sentiment analysis with online reviews is still relatively scarce in the existing research, directly or indirectly.

Zhang et al. [46] presented a feature-based method to rank alternatives with thousands of online reviews. In this method, alternative features would be extracted based on the frequencies and relative usage. The sentiment analysis method was used to identify the positive or negative sentiment orientation of each subjective sentence and each comparative sentence. The ranking of alternatives was determined based on the weighted and directed graph. Considering that different reviews have different levels of importance, Zhang et al. [47] improved the method proposed above. In this improved model, the ranking of alternatives was finally determined by the weights of alternative review factors, mainly including posting time and its helpfulness votes. And the ranking of alternatives still only depends on the positive and negative orientations in the reviews. Peng et al. [48] firstly applied the fuzzy decision-making method to rank alternatives through online reviews, and proposed a fuzzy PROMETHEE method. In this study, key alternative features were extracted by using tokenization and POS tagging on online reviews. A fuzzy evaluation matrix on several alternatives with several different significant features was obtained according to the subjective scoring from related domain experts. Finally, the ranking of alternatives was determined by the fuzzy PROMETHEE method. Similar studies also include [49-52] etc. To sum up, the above studies can be summarized as the alternatives ranking problem based on binary sentiment classification (i.e., positive and negative). In other words, the neutral sentiment information in online reviews is ignored in the final ranking determination. Meanwhile, most of the above studies do not realize the strong ambiguity and uncertainty of online reviews.

To overcome the limitations in the above research, Liu et al. [8] proposed the first study to combine the sentiment analysis method with IFS theory for handling neutral sentiment information in online reviews. Later, several scholars have carried out a series of related research on this basis. Liu et al. [53] pointed out that the difference in the number of online reviews of different alternatives would likely affect the final ranking result of alternatives, and the interval-valued IF-TOPSIS method was used to solve this problem. Bi et al. [54] considered the limited accuracy rates of the sentiment classification, which would affect the determination of the final ranking result. To this end, the interval type-2 fuzzy numbers were constructed to make up 
for this limitation. Yang et al. [55] emphasized that online consumers tend to have a habit of giving more weight to recent review information and less weight to earlier, while the existing research ignores it. As a result, the dynamic information preferences of consumers should be considered for the final ranking. Cali and Balaman [56] pointed out that determining the weights of criteria subjectively would become quite difficult when the number of criteria is too much. Therefore, the objective weighting based on the entropy method can avoid this problem very well, and it can handle the ambiguity and uncertainty in reviews as well. In addition, some scholars proposed other ways to convert the results of sentiment analysis into IFNs. For example, Wu and Zhang [57] and Zhang et al. [58] invited domain experts to determine the emotional intensity of each word using IFNs. The sentiment score was calculated for each feature in each review based on the sentiment analysis techniques. Finally, the corresponding overall sentiment score of each feature was obtained by using the intuitionistic fuzzy weighted averaging operator. Liang and Wang [59] converted the results of sentiment analysis into linguistic intuitionistic fuzzy numbers. Based on the cloud model, the information of linguistic membership and nonmembership degree was depicted by using three numerical characters, including expectation $E x$, entropy En and hyper entropy $\mathrm{He}$. Finally, the IFNs based on the normal clouds were constructed.

Later, a novel method to rank alternatives through online reviews based on sentiment analysis and HFS theory was proposed [58]. This work provides a new perspective to rank alternatives through online reviews. However, in this method, the hesitant fuzzy numbers (HFNs) of each feature of each alternative were constructed by domain experts, so there was strong subjectivity in it. To this end, this paper extends the identification of criteria of alternatives to the sub-criteria layer, and constructs HFNs objectively based on the intuitionistic fuzzy information. Besides, some other extensions of fuzzy sets have been widely applied to solve such problems in recent years, such as the probabilistic linguistic term sets [60], spherical hesitant fuzzy sets [61] and probabilistic hesitant fuzzy sets [62]. As a result, Table 1 summarizes some of the notable works in this domain.

\section{The Problem of Ranking TAs Through Online Reviews}

The main goal of this paper is to develop a RS for the potential tourist who tends to look through online reviews from experienced tourists before the journey. The problem of ranking TAs through online reviews is vividly described in Fig. 2. When a potential tourist has a desire to travel somewhere, some possible alternatives are usually determined through a preliminary investigation. However, the potential tourist is hesitant to make a choice between several alternatives due to the limited knowledge and expertise. Therefore, the potential tourist will choose to read online reviews related to the alternatives from the experienced tourists through the OTWs. Unfortunately, it is nearly impossible for the potential tourist to analyze the massive online reviews in a short period of time. To this end, how to extract important and useful information from the tremendous amount of online reviews and then rank alternative TAs is the problem that is addressed in this study. In general, the potential tourist wants to make the most comprehensive assessment and analysis for alternative TAs from various aspects, but usually ignores some important aspects due to the limited cognition, and results in decision-making mistakes. Therefore, extracting the aspects that the experienced tourists concern from a tremendous amount of online reviews can greatly help the potential tourist to have a deeper and more comprehensive understanding of the TAs, and reduce the individual's decision-making risk as well. When the aspects that experienced tourists concern are extracted from online reviews related to the alternatives, the collective preferences from experienced tourists on various aspects should be considered. At the same time, the potential tourist tends to have individual preferences for some aspects. As a result, the individual preference information for aspects is offered by the potential tourist. Then, these preferences are integrated to determine the weights of aspects. Finally, the ranking list of alternative TAs is obtained using a MCDM method to provide support in the potential tourist's decision-making.

\section{The Methodology}

The proposed RS in this paper consists of four phases. The first phase is to crawl online reviews of alternative TAs from OTWs by using web crawler software. Next, we extract the aspects that the experienced tourists concern from online reviews to construct a three-level evaluation system (including target layer, criteria layer and sub-criteria layer). Utilizing aspect-level sentiment analysis to identify the sentiment orientations (i.e., positive, neutral or negative) of sub-criteria through Baidu AI platform, where a HF-decision matrix is constructed based on IFS theory and HFS theory. Then, the preference information from the potential tourist and experienced tourists is integrated to determine the weights of criteria. The last phase is to use a MCDM method, the IHF-TOPSIS, to rank the alternative TAs. The process of the proposed methodology is shown in Fig. 3. 
Table 1 The notable works in ranking alternatives through online reviews

\begin{tabular}{|c|c|}
\hline References & Contents \\
\hline Zhang et al. [47] & Ranking score calculation for products based on the importance of review information \\
\hline Peng et al. [48] & Mining customer reviews based on the fuzzy PROMETHEE approach \\
\hline Liu et al. [8] & Ranking products through online reviews based on sentiment analysis and intuitionistic fuzzy set theory \\
\hline Liu et al. [53] & $\begin{array}{l}\text { Ranking products through online reviews based on sentiment analysis and interval-valued intuitionistic fuzzy TOPSIS } \\
\text { approach }\end{array}$ \\
\hline $\begin{array}{l}\text { Cali and Balaman } \\
\text { [56] }\end{array}$ & Combining sentiment analysis and intuitionistic fuzzy multi-criteria evaluation to support decision making \\
\hline Zhang et al. [58] & Ranking products through online reviews based on hesitant fuzzy set and sentiment analysis \\
\hline Luo et al. [60] & $\begin{array}{l}\text { Tourism attraction selection with online reviews by using probabilistic linguistic term sets and the IDOCRIW-COCOSO } \\
\text { model }\end{array}$ \\
\hline Khan et al. [61] & A decision support model for hotel recommendation through logarithmic spherical hesitant fuzzy information \\
\hline Cao et al. [62] & $\begin{array}{l}\text { A probabilistic hesitant fuzzy recommendation decision-making algorithm based on bipartite network projection for } \\
\text { selecting sharing accommodation }\end{array}$ \\
\hline Liang et al. [63] & $\begin{array}{l}\text { A data-driven decision-making analysis model based on probabilistic linguistic term sets and online reviews for web } \\
\text { celebrity shop assessment and improvement }\end{array}$ \\
\hline Liang et al. [64] & $\begin{array}{l}\mathrm{O} 2 \mathrm{O} \text { takeaway customer satisfaction evaluation based on online reviews by combining fuzzy comprehensive evaluation } \\
\text { with AHP and probabilistic linguistic item sets }\end{array}$ \\
\hline
\end{tabular}

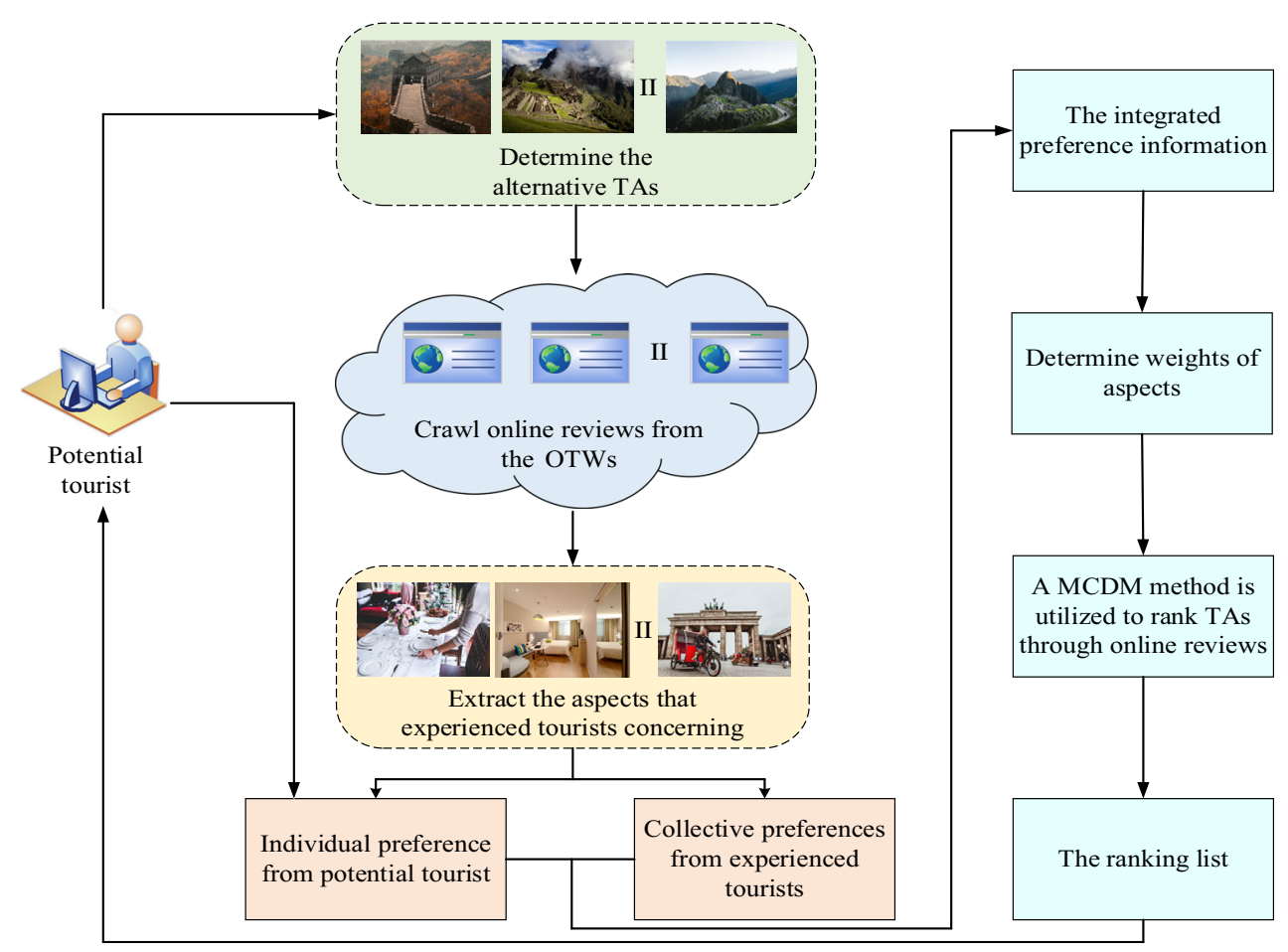

Fig. 2 The problem of ranking TAs through online reviews

\subsection{Measuring Sentiment Orientations of Online Reviews}

According to the related work in Sect. 2.1, the sentiment analysis method based on deep learning has a superior performance in sentiment classification compared to traditional methods. At the same time, in order to extract the aspects that the experienced tourists concern in online reviews, the aspect-level sentiment analysis based on deep learning is applied to this paper. For this, three parts are included in this phase: (1) web crawler technique-based Python software is used to crawl and preprocess online reviews on TAs; (2) Baidu AI platform is used to extract the aspects that the experienced tourists concern and 


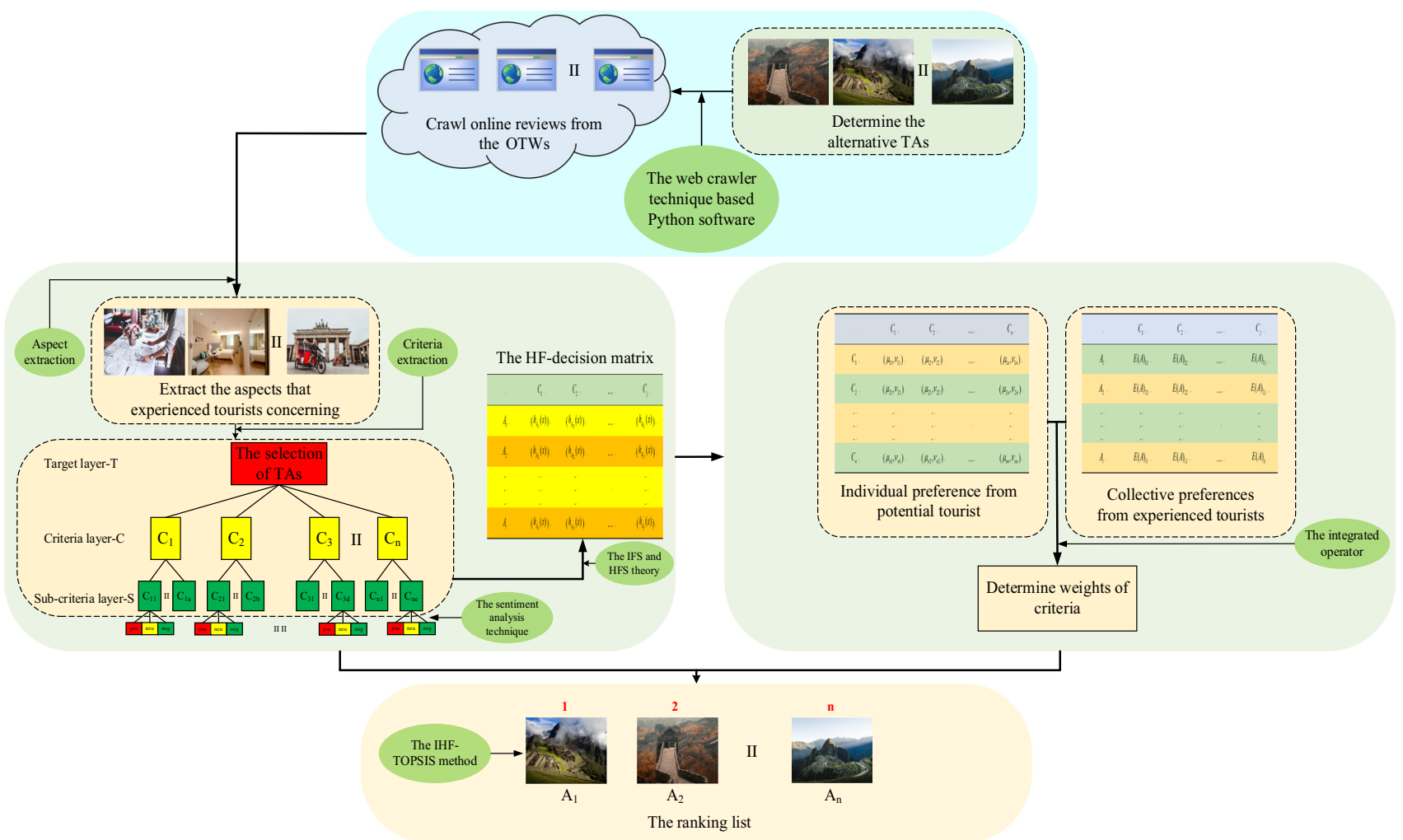

Fig. 3 The process of proposed methodology

calculate the sentiment orientation; (3) the results of aspect-level sentiment analysis are converted to IFNs and HFNs.

\subsubsection{Data Crawling and Preprocessing}

In this paper, online reviews on TAs are crawled from the related OTWs by using Python software. Based on the Xpath (XML Path Language) technique, online reviews are quickly located in the web page so as to achieve the bulk acquisition of data. When we get an original dataset about online reviews from web pages, there are often full of many duplicate reviews, blank reviews and irregular characters. Therefore, it is necessary to perform data preprocessing. In the process of data preprocessing, duplicate and blank reviews are deleted, and irregular characters are regularization processed by Python software. Also, reviews with no more than ten words are eliminated. More importantly, the necessary manual checks are performed in order to ensure the authenticity and relevance of reviews.

\subsubsection{Aspect Extraction and Calculating the Sentiment Orientation}

In this subsection, we aim to extract the aspects (i.e., criteria) that experienced tourists concern with alternatives, and build a three-level evaluation system (including target layer, criteria layer and sub-criteria layer) and calculate the sentiment orientations of sentences for each sub-criterion.

In general, one or a few sentences are included in an online review to express the opinion on the TA concerning several aspects. Therefore, the proposed methodology regards sentences as a measurement unit of reviews. If several sentences are included in an online review, it will be split into several separate sentences according to the punctuation marks in the review. For example, in an online review, "I think the traffic in this tourist attraction is very convenient, but I am not satisfied with the environment.", "traffic" and "environment" two aspects are included, and it is further split into two separate sentences: "I think the traffic in this tourist attraction is very convenient" and "But I am not satisfied with the environment.".

In a sentence, two parts are usually included, which are focus point and opinion. The focus point is considered to determine the important aspect of alternatives and the opinion reflects the sentiment orientation of tourists on this aspect. After splitting online reviews into separate sentences, the focus point and opinion are easily extracted through calling the function of opinion extraction in the Baidu AI platform (https://ai.baidu.com/docs\#/NLPPython-SDK/5b7e7f23). For instance, Fig. 4 shows a result of the aspect extraction. In the sentence 




Fig. 4 An example of the aspect extraction

我觉得这个景区的交通很便利. (i.e. “I think the traffic in this tourist attraction is very convenient."), the focus point "traffic" and the opinion "convenient" are extracted. Besides, the aspect-level sentiment classification result is given, and the number 0 means negative, the number 1 means neutral and the number 2 means positive, which greatly simplifies subsequent work. In Fig. 4, this sentence belongs to a positive classification, and it further verifies the accuracy of the sentiment classification algorithm. After extracting the focus point of each sentence in online reviews, the focus points with high frequency are considered to determine the important aspects of alternative TAs. In order to evaluate the TA from various aspects and simplify the decision-making process as well, we summarize and group the fine-grained aspects that belong to the same category. As a result, a three-level evaluation system is constructed, shown in Fig. 5. Finally, the sentiment orientations of sentences for each sub-criterion are calculated.

\subsubsection{Converting the Result of Aspect-Level Sentiment Analysis to IFNs and HFNs}

The HFS introduced by Torra [24] can accurately reflect the ambiguity and uncertainty in practical decision-making problems. The HFNs are composed of several possible degrees of membership, which is generally expressed as $h=h_{A}(x)$ [34]. In the three-level evaluation system, each criterion consists of several sub-criteria. Therefore, the

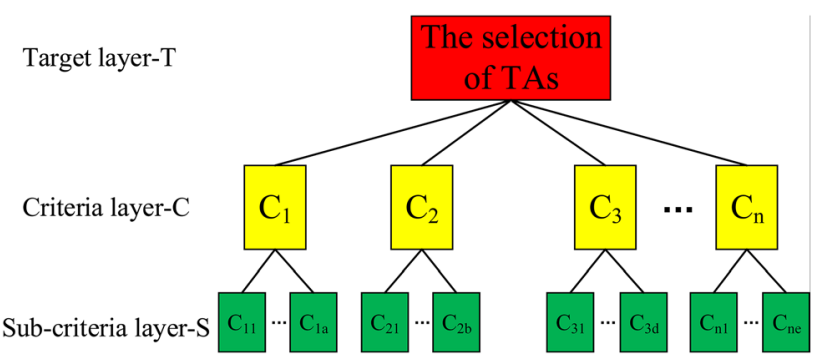

performance score of each criterion of each alternative is represented by HFNs.

Let $A=\left\{A_{1}, A_{2}, \cdots, A_{n}\right\}$ be a set of alternatives, where $A_{i}$ denotes the ith alternative, $i=1,2, \cdots, n ; \quad C=$ $\left\{C_{1}, C_{2}, \cdots, C_{m}\right\}$ be a set of criteria of alternatives, where $C_{j}$ denotes the $j$ th criterion, $j=1,2, \cdots, m ; S=\left\{S_{1}, S_{2}, \cdots\right.$ $\left.\cdot, S_{a}\right\}$ be a set of sub-criteria of alternatives, where $S_{k}$ denotes the $k t h$ sub-criterion, $k=1,2, \cdots, a$. First of all, the number of the positive $N_{i k}^{2}$, neutral $N_{i k}^{1}$ and negative $N_{i k}^{0}$ sentences concerning each sub-criterion of each alternative is calculated. As a result, the percentages $P_{i k}$ of the positive, neutral and negative sentences related to each subcriterion of each alternative are obtained as follows:

$P_{i k}^{2}=\frac{N_{i k}^{2}}{N_{i k}^{2}+N_{i k}^{1}+N_{i k}^{0}}$

$P_{i k}^{1}=\frac{N_{i k}^{1}}{N_{i k}^{2}+N_{i k}^{1}+N_{i k}^{0}}$

$P_{i k}^{0}=\frac{N_{i k}^{0}}{N_{i k}^{2}+N_{i k}^{1}+N_{i k}^{0}}$

where $P_{i k}^{2}+P_{i k}^{1}+P_{i k}^{0}=1$ and $P_{i k}^{2}, P_{i k}^{1}, P_{i k}^{0} \in[0,1][0,1]$.

The membership degree $\mu_{i k}=P_{i k}^{2}$, non-membership degree $\pi_{i k}=P_{i k}^{0}$ and hesitant degree $v_{i k}=P_{i k}^{1}$, which denote the satisfaction, dissatisfaction and neutral degrees of experienced tourists, respectively. As a result, the converted intuitionistic fuzzy information of each sub-criterion concerning each alternative is acquired based on IFS theory [56]. The performance score of each criterion of each alternative is denoted as:

$h_{i j}=\left(\mu_{i 1}, \mu_{i 2}, \cdots, \mu_{i t}\right)$

where $\mu_{i t}$ denotes the membership degree of the tth subcriterion contained in the $j$ th criterion.

Furthermore, the HFN $h_{i j}$ contains several possible degrees of membership, which fully reflects the hesitation information of experienced tourists, and it also avoids that

Fig. 5 The three-level evaluation system 
the decision-making process is too complicated due to too many sub-criteria.

\subsection{Determining Weights of Criteria Based on Preference Information}

In this subsection, individual preferences from the potential tourist and collective preferences from experienced tourists together determine the weights of criteria so that the final ranking result is more in line with the actual situation. Therefore, the IFPR is used to extract individual preferences and HF-entropy is used to extract collective preferences. The optimal integrated weights of criteria are obtained based on the principle of deviation minimization.

\subsubsection{Individual Preferences from the Potential Tourist}

The problem of MCDM is considered as using a set of the evaluation system to choose the best alternative or rank alternatives. Hence, the decision-maker tends to have his/her own subjective preference for each criterion. In this paper, the IFPR is used to depict the preference of the potential tourist on different criteria. Table 2 shows an IFPR $R=\left(r_{j j^{\prime}}\right)_{m \times m}$.

A typical fractional programming method is used to derive the precise priority weight of each criterion from the IFPR. Figure 6 shows the deterministic process of priority weight.

After obtaining the IFPR from the decision-maker, the multiplicative consistency needs to be satisfied usually, then the priority weight vector is determined. When it does not satisfy the multiplicative consistency, the model can also derive the weight vector. Furthermore, if the decision-maker believes that the result meets the expected preference, the calculation process is also completed. Otherwise, the decision-maker should reevaluate each criterion until constructing an acceptable IFPR for multiplicative consistency.

Definition 1 [65] The IFPR $R=\left(r_{j j^{\prime}}\right)_{m \times m}$ satisfies the multiplicative consistency, if

$\mu_{j j^{\prime}} \cdot \mu_{j^{\prime} t} \cdot \mu_{t j}=v_{j j^{\prime}} \cdot v_{j^{\prime} t} \cdot v_{t j}, j, t, j^{\prime}=1,2, \cdots, m$

Let $\omega=\left(\omega_{1}, \omega_{2}, \cdots, \omega_{j}\right)^{T}$ be the priority weight vector of

Table 2 The IFPR matrix $R=\left(r_{j j^{\prime}}\right)_{m \times m}$

\begin{tabular}{lllll}
\hline & $C_{1}$ & $C_{2}$ & $\ldots$ & $C_{m}$ \\
\hline$C_{1}$ & $\left(\mu_{11}, v_{11}\right)$ & $\left(\mu_{12}, v_{12}\right)$ & $\ldots$ & $\left(\mu_{1 m}, v_{1 m}\right)$ \\
$C_{2}$ & $\left(\mu_{21}, v_{21}\right)$ & $\left(\mu_{22}, v_{22}\right)$ & $\ldots$ & $\left(\mu_{2 m}, v_{2 m}\right)$ \\
$\vdots$ & $\vdots$ & $\vdots$ & & $\vdots$ \\
$C_{m}$ & $\left(\mu_{m 1}, v_{m 1}\right)$ & $\left(\mu_{m 2}, v_{m 2}\right)$ & $\ldots$ & $\left(\mu_{m m}, v_{m m}\right)$
\end{tabular}

where $\mu_{i j^{\prime}}$ denotes the preference degree of the criterion $j$ for the criterion $j^{\prime} ; v_{i j j^{\prime}}$ denotes the preference degree of the criterion $j^{\prime}$ for the criterion $j$ criteria derived from the IFPR and $\sum_{j=1}^{m} \omega_{j}=1$. To simultaneously display the situation of the multiplicative consistency and inconsistency, the slack variables $\psi_{j j^{\prime}}$ and $\zeta_{j j^{\prime}}$ are introduced, which satisfies:

$$
\begin{gathered}
\mu_{j j^{\prime}}-\psi_{j j^{\prime}} \leq \frac{\omega_{j}}{\omega_{j}+\omega_{j^{\prime}}} \leq 1-v_{j j^{\prime}}+\zeta_{j j^{\prime}}, j \\
\quad=1,2, \cdots, m-1 ; j^{\prime}=j+1, \cdots, m
\end{gathered}
$$

where $\psi_{j j^{\prime}} \geq 0, \zeta_{j j^{\prime}} \geq 0$ and $\psi_{j j^{\prime}} \cdot \zeta_{j j^{\prime}}=0$.

When the IFPR satisfies the multiplicative consistency, $\psi_{j j^{\prime}}=\zeta_{j j^{\prime}}=0$; when the IFPR does not satisfy the multiplicative consistency, $\psi_{j j^{\prime}}=0$ or $\zeta_{j j^{\prime}}=0$. Therefore, the priority weight vector $\omega$ is calculated by the following nonlinear programming model:

$$
\begin{aligned}
& \min f=\sum_{j=1}^{m-1} \sum_{j^{\prime}=j+1}^{m}\left(\psi_{j j^{\prime}}+\zeta_{j j^{\prime}}\right) \\
& \text { s.t. }\left\{\begin{array}{l}
\frac{\omega_{j}}{\omega_{j}+\omega_{j^{\prime}}}+\psi_{j j^{\prime}}-\mu_{j j^{\prime}} \geq 0, j=1,2, \cdots, m-1 ; j^{\prime}=j+1, \cdots, m \\
\frac{\omega_{j}}{\omega_{j}+\omega_{j^{\prime}}}-\zeta_{j j^{\prime}}+v_{j j^{\prime}} \leq 1, j=1,2, \cdots, m-1 ; j^{\prime}=j+1, \cdots, m \\
\sum_{j=1}^{m} \omega_{j}=1, \omega_{j} \in[0,1], j=1,2, \cdots, m \\
\psi_{j j^{\prime}} \geq 0, \zeta_{j j^{\prime}} \geq 0, \psi_{j j^{\prime}} \cdot \zeta_{j j^{\prime}}=0, j=1,2, \cdots, m-1 ; j^{\prime}=j+1, \cdots, m
\end{array}\right.
\end{aligned}
$$

According to the optimal solution $f$, the multiplicative consistency of the IFPR is judged.

Definition 2 [66]. The IFPR satisfies the multiplicative consistency if and only if the optimal solution of the objective function $f^{*}=0$.

\subsubsection{Collective Preferences From Experienced Tourists}

According to the explanation in information theory [67], the concept of entropy is an indicator for measuring the disorder degree of the system. Zeleny [68] firstly confirmed that Shannon's entropy measure is an effective tool to determine the relative importance of each criterion (i.e., weight). Later, it is widely recognized by scholars. In the evaluation system, if the entropy value of a criterion is smaller, the less information it contains, and the smaller the contribution to the final evaluation result, and the smaller weight should be. In this paper, the HF-entropy measure developed by [69] is used to determine the weights of criteria. The calculation process is as follows:

First, according to the HF-decision matrix $H=\left(h_{i j}\right)_{n \times m}$, the HF-entropy measure of each HFN $h_{i j}$ is calculated by Eq. (8): 


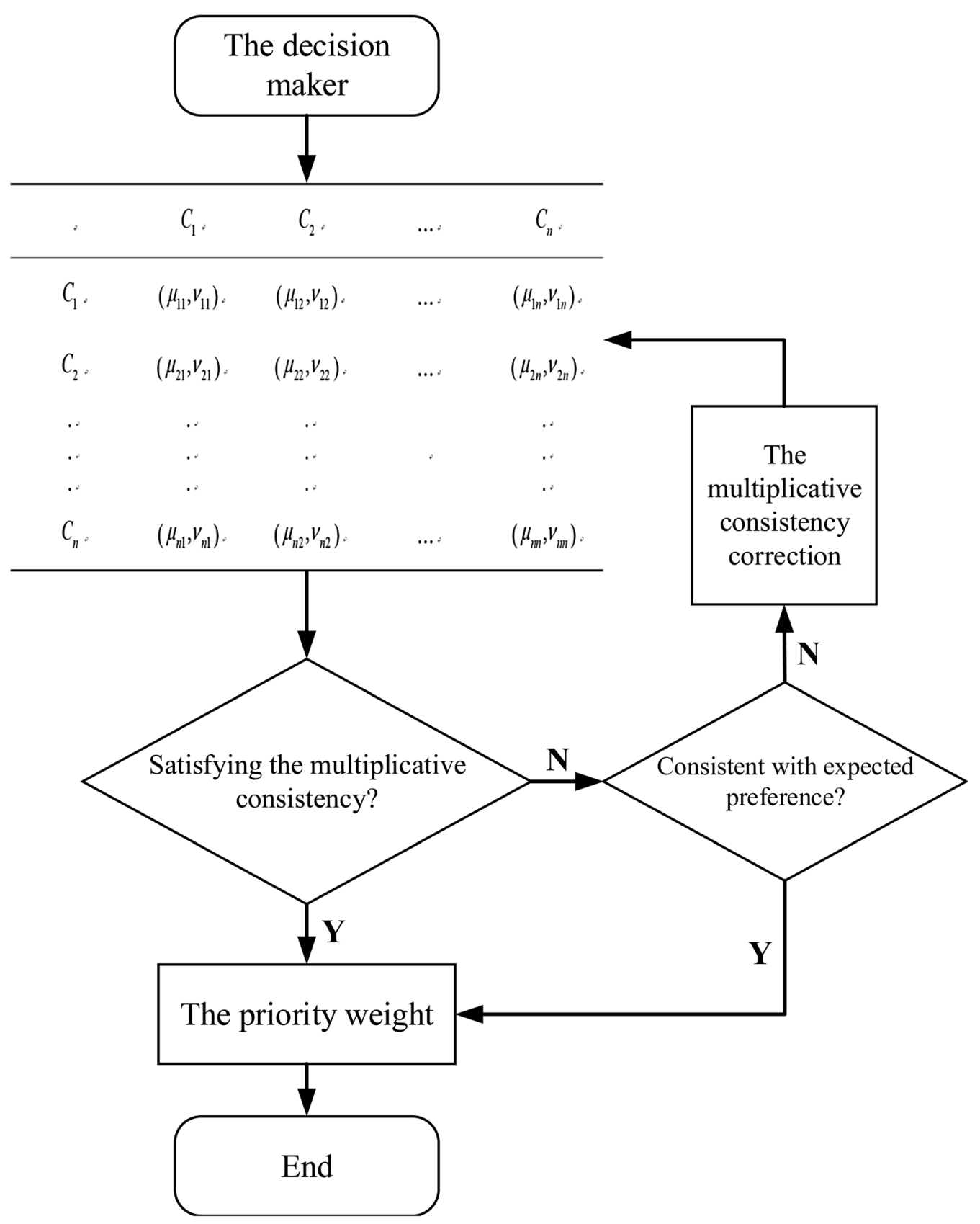

Fig. 6 The deterministic process of priority weight

$E\left(h_{i j}\right)=-\frac{1}{l_{h} \ln 2} \sum_{g=1}^{l_{h}}\left(\begin{array}{r}\frac{h^{\sigma(g)}+h^{\sigma\left(l_{h}-g+1\right)}}{2} \ln \frac{h^{\sigma(g)}+h^{\sigma\left(l_{h}-g+1\right)}}{2} \\ +\frac{2-h^{\sigma(g)}+h^{\sigma\left(l_{h}-g+1\right)}}{2} \ln \frac{2-h^{\sigma(g)}+h^{\sigma\left(l_{h}-g+1\right)}}{2}\end{array}\right)$

where $l_{h}$ is the number of elements in $h$ and $g=1,2, \cdots, l_{h}$.

Then the entropy weight of each criterion is obtained by Eq. (9):
$\varpi_{j}=\frac{1-E_{j}}{n-\sum_{j=1}^{m} E_{j}}, j=1,2, \cdots, m$

where $E_{j}=\frac{1}{n} \sum_{i=1}^{n} E\left(h_{i j}\right), j=1,2, \cdots, m$ and $\sum_{j=1}^{m} \varpi_{j}=1$.

Finally, the priority weight vector of each criterion $\varpi=$ $\left(\varpi_{1}, \varpi_{2}, \cdots, \varpi_{j}\right)^{T}$ is obtained. The HF-entropy weight method is an objective weighting method that derives weights from raw data, which can be considered as the preferences of experienced tourists for each criterion in this paper. 


\subsubsection{Calculating the Final Weights of Criteria}

Since there are two sets of weights (subjective and objective weights from the potential tourist and experienced tourists, respectively), it is necessary for a final decisionmaking to integrate these weights into one single set [70]. In order to make full use of their respective advantages, an optimization model based on the principle of deviation minimization is introduced to solve this problem [71]. The calculation steps are as follows:

Step 1 Calculate $\omega_{j}^{1}$ and $\omega_{j}^{2}$ with the weight vector $\omega=$ $\left(\omega_{1}, \omega_{2}, \cdots, \omega_{j}\right)^{T}$ and $\varpi=\left(\varpi_{1}, \varpi_{2}, \cdots, \varpi_{j}\right)^{T}$, respectively: $\omega_{j}^{1}=\frac{\omega_{j} \times \varpi_{j}}{\sum_{j=1}^{m} \omega_{j} \times \varpi_{j}}$

$\omega_{j}^{2}=\frac{1}{2}\left(\omega_{j}+\varpi_{j}\right)$

Then, the combination weight vector $W^{1}=\left(\omega_{1}^{(1)}, \omega_{2}^{(1)}, \ldots\right.$ $\left.\cdot, \omega_{m}^{(1)}\right)$ and $W^{2}=\left(\omega_{1}^{(2)}, \omega_{2}^{(2)}, \cdots, \omega_{m}^{(2)}\right)$ are obtained. Step 2 Construct the optimal combination weight vector $W^{*}=\left(\omega_{1}^{*}, \omega_{2}^{*}, \cdots, \omega_{m}^{*}\right)$, which satisfies $\sum_{j=1}^{m} \omega_{j}^{*}=1, j=$ $1,2, \cdots, m$, where $\omega_{j}^{*}=\lambda_{1} \omega_{j}^{(1)}+\lambda_{2} \omega_{j}^{(2)}, j=1,2, \cdots, m$, then

$$
\begin{aligned}
& W^{*}-W^{1}=\left(\omega_{1}^{*}-\omega_{1}^{(1)}, \omega_{2}^{*}-\omega_{2}^{(1)}, \cdots, \omega_{m}^{*}-\omega_{m}^{(1)}\right) \\
& W^{*}-W^{2}=\left(\omega_{1}^{*}-\omega_{1}^{(2)}, \omega_{2}^{*}-\omega_{2}^{(2)}, \cdots, \omega_{m}^{*}-\omega_{m}^{(2)}\right)
\end{aligned}
$$

Step 3 Based on the principle of deviation minimization, the nonlinear optimization model is constructed as follows:

$\left\{\begin{array}{l}\min \sum_{k=1}^{2}\left\|W^{*}-W^{k}\right\|=\min \sum_{k=1}^{2} \sum_{j=1}^{m}\left(\omega_{j}^{*}-\omega_{j}^{k}\right)=\min \sum_{k=1}^{2} \sum_{j=1}^{m}\left(\lambda_{1} \omega_{j}^{(1)}+\lambda_{2} \omega_{j}^{(2)}-\omega_{j}^{(k)}\right)^{2} \\ \text { s.t. } \sum_{j=1}^{m} \omega_{j}=1\end{array}\right.$

Step 4 Construct the Lagrangian function:

$$
\begin{aligned}
L\left(\lambda_{1}, \lambda_{2}, M\right)= & \sum_{k=1}^{2} \sum_{j=1}^{m}\left(\lambda_{1} \omega_{j}^{(1)}+\lambda_{2} \omega_{j}^{(2)}-\omega_{j}^{(k)}\right)^{2} \\
& +M\left(\sum_{j=1}^{m} \omega_{j}-1\right)
\end{aligned}
$$

Then, the first-order partial derivative of $\lambda_{1}, \lambda_{2}, M$ is obtained:

$$
\text { Let }\left\{\begin{array}{l}
\frac{\partial L}{\partial \lambda_{1}}=0 \\
\frac{\partial L}{\partial \lambda_{2}}=0, \text { the optimal matrix representation is } \\
\frac{\partial L}{\partial M}=0
\end{array}\right.
$$

$\left[\begin{array}{l}W^{(1)}\left(W^{(1)}\right)^{T}+\lambda_{1} W^{(1)}\left(W^{(2)}\right)^{T} \\ W^{(2)}\left(W^{(1)}\right)^{T}+\lambda_{2} W^{(2)}\left(W^{(2)}\right)^{T}\end{array}\right]\left[\begin{array}{l}\lambda_{1} \\ \lambda_{2}\end{array}\right]=\left[\begin{array}{l}W^{(1)}\left(W^{(1)}\right)^{T} \\ W^{(2)}\left(W^{(2)}\right)^{T}\end{array}\right]$

Then, we transform it into a form of equations:

$\left\{\begin{array}{l}\lambda_{1} W^{(1)}\left(W^{(1)}\right)^{T}+\lambda_{2} W^{(1)}\left(W^{(2)}\right)^{T}=W^{(1)}\left(W^{(1)}\right)^{T} \\ \lambda_{1} W^{(2)}\left(W^{(1)}\right)^{T}+\lambda_{2} W^{(2)}\left(W^{(2)}\right)^{T}=W^{(2)}\left(W^{(2)}\right)^{T}\end{array}\right.$

Step 5 Obtain the values of $\lambda_{1}$ and $\lambda_{2}$. As a result, the optimal combination weight vector $W^{*}=\left(\omega_{1}^{*}, \omega_{2}^{*}, \cdot\right.$ $\cdot,\left(\omega_{m}^{*}\right)^{T}$ is calculated.

\subsection{Ranking TAs Using the IHF-TOPSIS Method}

The final phase of the proposed RS is to rank alternative TAs based on the performance score of each criterion of each alternative. In this paper, the IHF-TOPSIS method is employed, which is described in detail in Subsection 4.3.2.

\subsubsection{Definition and operations of HFSs}

Before introducing the method of IHF-TOPSIS, we first recall some concepts including definition and operations related to HFSs.

Definition 3 [34]. Let $X$ be a fixed set, the hesitant fuzzy sets (HFS) on $X$ can be expressed as:

$A=\left\{\left\langle x, h_{A}(x)\right\rangle \mid x \in X\right\}$

where $h_{A}(x)$ is a set of some values in [0,1], denoting the possible membership degrees of the element $x \in X$ to the set $A$.

For convenience, $h=h_{A}(x)$ is also called hesitant fuzzy element (HFE) [72].

Definition 4 [73]. Let $h, h_{1}$ and $h_{2}$ be the three HFEs, then some operations on them can be represented as:

(1) $h^{\lambda}=\cup_{\gamma \in h}\left\{\gamma^{\lambda}\right\}$;

$$
\begin{aligned}
& \text { (2) } \lambda h=\underset{\gamma \in h}{\cup}\left\{1-(1-\gamma)^{\lambda}\right\} ; \\
& \text { (3) } h_{1} \oplus h_{2}=\underset{\substack{\gamma_{1} \in h_{1} \\
\gamma_{2} \in h_{2}}}{\cup}\left\{\gamma_{1}+\gamma_{2}-\gamma_{1} \gamma_{2}\right\} ; \\
& \text { (4) } h_{1} \otimes h_{2}=\underset{\substack{\gamma_{1} \in h_{1} \\
\gamma_{2} \in h_{2}}}{\bigcup}\left\{\gamma_{1} \gamma_{2}\right\} .
\end{aligned}
$$

Definition 5. Let $h_{i}(i=1,2, \cdots, n)$ be a set of HFEs, the hesitant fuzzy-weighted averaging (HFWA) operator can be expressed as: 


$$
\begin{aligned}
\operatorname{HFWA}\left(h_{1}, h_{2}, \cdots, h_{n}\right)= & \bigoplus_{i=1}^{n} \omega_{i} h_{i} \\
= & \bigcup_{\gamma_{1} \in h_{1}, \gamma_{2} \in h_{2}, \cdots, \gamma_{n} \in h_{n}}\{1 \\
& \left.-\prod_{i=1}^{n}\left(1-\gamma_{i}\right)^{\omega_{i}}\right\}
\end{aligned}
$$

where $\omega=\left(\omega_{1}, \omega_{2}, \cdots, \omega_{n}\right)^{T}$ is the weight vector of $h_{i}(i=1,2, \cdots, n), \quad \omega_{i} \in[0,1], i=1,2, \cdots, n \quad$ and $\sum_{i=1}^{n} \omega_{i}=1$. Particularly, if $\omega=\left(\frac{1}{n}, \frac{1}{n}, \cdots, \frac{1}{n}\right)^{T}$, then the HFWA operator degenerates to the hesitant fuzzy averaging (HFA) operator:

$$
\begin{aligned}
H W A\left(h_{1}, h_{2}, \cdots, h_{n}\right) & =\frac{1}{n} \bigoplus_{i=1}^{n} h_{i} \\
& =\underset{\gamma_{1} \in h_{1}, \gamma_{2} \in h_{2}, \cdots, \gamma_{n} \in h_{n}}{\cup}\left\{1-\prod_{i=1}^{n}\left(1-\gamma_{i}\right)^{\frac{1}{n}}\right\}
\end{aligned}
$$

Definition 6 [37]. Assume that there are two HFEs $A_{1}$ and $A_{2}$, the Euclidean distance between them is calculated as:

$d=\left(A_{1}, A_{2}\right)=\sqrt{\frac{1}{l_{x}} \sum_{j=1}^{l_{x}}\left|h_{A_{1}}^{\sigma(q)}(x)-h_{A_{2}}^{\sigma(q)}(x)\right|^{2}}$

where $h_{A_{1}}^{\sigma(q)}(x)$ and $h_{A_{2}}^{\sigma(q)}(x)$ are the $q$ th largest value of $h_{A_{1}}(x)$ and $h_{A_{2}}(x)$, respectively.

\subsubsection{IHF-TOPSIS Method}

Technique for Order Performance by Similarity to Ideal Solution (TOPSIS) is a typical evaluation ranking method applicable to a limited number of evaluation objects with multiple criteria or attributes, firstly proposed by Hwang and Yoon [74]. Compared with other evaluation methods, the TOPSIS method does not have strict requirements on the number of criteria and data distribution, so the original data in the evaluation system can be fully utilized, which greatly reduces the loss of evaluation information [75, 76]. In recent years, this method has been effectively extended to the MCDM problems under fuzzy environment [77-79].

The steps of the IHF-TOPSIS method are outlined as follows:

Step 1. Obtain the intuitionistic fuzzy information for sub-criteria.

Table 3 The HF-decision matrix $H=\left(h_{i j}\right)_{n \times m}$

\begin{tabular}{ccccc}
\hline & $C_{1}$ & $C_{2}$ & $\ldots$ & $C_{m}$ \\
\hline$A_{1}$ & $h_{11}$ & $h_{12}$ & $\ldots$ & $h_{1 m}$ \\
$A_{2}$ & $h_{21}$ & $h_{22}$ & $\ldots$ & $h_{2 m}$ \\
$\vdots$ & $\vdots$ & $\vdots$ & & $\vdots$ \\
$A_{n}$ & $h_{n 1}$ & $h_{n 2}$ & $\ldots$ & $h_{n m}$ \\
\hline
\end{tabular}

The opinioned sentences for each sub-criterion are transformed into intuitionistic fuzzy information (i.e., membership, hesitant and non-membership degree) based on the aspect-level sentiment analysis, which is described in detail in Subsection 4.1.3.

Step 2. Construct the HF-decision matrix.

Let $A=\left\{A_{1}, A_{2}, \cdots, A_{n}\right\}$ be optional alternatives, $C=$ $\left\{C_{1}, C_{2}, \cdots, C_{m}\right\}$ be the criterion of each alternative. Based on intuitionistic fuzzy information, the performance scores of criteria of alternatives are constructed by HFNs, and the HF-decision matrix $H=\left(h_{i j}\right)_{n \times m}$ is shown in Table 3 .

Step 3 Determine the weights of criteria.

According to the obtained evaluation criteria from online reviews, the individual preferences from the potential tourist for criteria are measured by the method described in Subsection Individual Preferences from the Potential Tourist. The collective preferences from experienced tourists for criteria are calculated based on the HFdecision matrix $H=\left(h_{i j}\right)_{n \times m}$ above, which is explained in detail in Subsection Collective Preferences From Experienced Tourists. Finally, the optimal combination weight $W^{*}=\left(\omega_{1}^{*}, \omega_{2}^{*}, \cdots, \omega_{m}^{*}\right)$ is obtained by the method described in Subsection Calculating the Final Weights of Criteria, where $\omega_{j}^{*} \in[0,1](j=1,2, \cdots, m)$ and $\sum_{j=1}^{m} \omega_{j}^{*}=1$.

Step 4 Construct the weighted HF-decision matrix.

Based on Eq. (21), the weighted HF-decision information is obtained:

$\bar{h}_{i j}=\omega_{j}^{*} h_{i j}=\cup_{\gamma \in h_{i j}}\left\{1-(1-\gamma)^{\omega_{j}^{*}}\right\}$

Then, the weighted HF-decision matrix $\bar{H}=\left(\bar{h}_{i j}\right)_{n \times m}$ is constructed as shown in Table 4.

Step 5 Determine the HF positive-ideal (HFPI) and HF negative-ideal (HFNI) solutions.

Let $A^{+}$be the HFPI solution and $A^{-}$be the HFNI solution, denoted as:

$A^{+}=\left\{\left\langle x_{j}, \max _{i}\left\{\bar{h}_{i j}^{\sigma(q)}\right\}\right\rangle \mid(j=1,2, \cdots, m)\right\}$
$A^{-}=\left\{\left\langle x_{j}, \min _{i}\left\{\bar{h}_{i j}^{\sigma(q)}\right\}\right\rangle \mid(j=1,2, \cdots, m)\right\}$

where $A_{m}^{+}=\left\{\left\langle x_{m},\left\{\left(h_{m}^{1}\right)^{+},\left(h_{m}^{2}\right)^{+}, \cdots,\left(h_{m}^{l}\right)^{+}\right\}\right\rangle\right\} \quad$ and $A_{m}^{-}=\left\{\left\langle x_{m},\left\{\left(h_{m}^{1}\right)^{-},\left(h_{m}^{2}\right)^{-}, \cdots,\left(h_{m}^{l}\right)^{-}\right\}\right\rangle\right\}$.
Table 4 The weighted HF-decision matrix $\bar{H}=\left(\bar{h}_{i j}\right)_{n \times m}$

\begin{tabular}{ccccc}
\hline & $C_{1}$ & $C_{2}$ & $\cdots$ & $C_{m}$ \\
\hline$A_{1}$ & $\bar{h}_{11}$ & $\bar{h}_{12}$ & $\ldots$ & $\bar{h}_{1 m}$ \\
$A_{2}$ & $\bar{h}_{21}$ & $\bar{h}_{22}$ & $\cdots$ & $\bar{h}_{2 m}$ \\
$\vdots$ & $\vdots$ & $\vdots$ & & $\vdots$ \\
$A_{n}$ & $\bar{h}_{n 1}$ & $\bar{h}_{n 2}$ & $\cdots$ & $\bar{h}_{n m}$ \\
\hline
\end{tabular}


Step 6 Calculate the distance between the alternative and the HFPI solution or the HFNI solution.

The distances are calculated using the Euclidean distance [38]:

$$
\begin{aligned}
D\left(A_{i}, A^{+}\right) & =\sum_{j=1}^{m} d\left(h_{i j}, h_{j}^{+}\right) \\
& =\sum_{j=1}^{m} \sqrt{\frac{1}{l} \sum_{q=1}^{l}\left|h_{i j}^{\sigma(q)}-\left(h_{j}^{\sigma(q)}\right)^{+}\right|^{2}}, i=1,2, \cdots, n
\end{aligned}
$$

$$
\begin{aligned}
D\left(A_{i}, A^{-}\right) & =\sum_{j=1}^{m} d\left(h_{i j}, h_{j}^{-}\right) \\
& =\sum_{j=1}^{m} \sqrt{\frac{1}{l} \sum_{q=1}^{l}\left|h_{i j}^{\sigma(q)}-\left(h_{j}^{\sigma(q)}\right)^{-}\right|^{2}}, i=1,2, \cdots, n
\end{aligned}
$$

Step 7 Calculate the relative closeness degree of each alternative to the HFPI solution.

The relative closeness degree $C_{i}$ of each alternative to the HFPI is calculated by Eq. (26).

$C_{i}=\frac{D\left(A_{i}, A^{-}\right)}{D\left(A_{i}, A^{+}\right)+D\left(A_{i}, A^{-}\right)}, i=1,2, \cdots, n$

where $0 \leq C_{i} \leq 1, i=1,2, \cdots, n$.

Step 8 Rank the alternatives.

The alternatives are ranked by the values of relative closeness degrees in descending order. Therefore, the decision-maker can choose the optimal alternative according to the final ranking result.

\section{A Case Study}

\subsection{Selecting the Optimal TA with the Proposed Methodology}

The RS proposed in this paper is aimed to rank the alternative TAs through online reviews based on the aspectlevel sentiment analysis and the IHF-TOPSIS method. For this, a case study is given to prove the validity of the proposed methodology. Considering a potential tourist who wants to travel somewhere, 5 alternative TAs are selected after a preliminary investigation, i.e.,

$A_{1}$ : The Palace Museum (Location: Beijing, China).

$A_{2}$ : The West Lake (Location: Zhejiang province, China).

$A_{3}$ : The Mount Huangshan (Location: Anhui province, China).

$A_{4}$ : The Mount Lu (Location: Jiangxi province, China).

$A_{5}$ : The Longmen Grottoes (Location: Henan province, China).
Firstly, online reviews of these TAs are crawled from Qunar in May 2019, which is an online travel agency (https://www.qunar.com) aimed to help Chinese tourists make better travel choices, mainly through collecting various information related to travel, including airfare, hotels, visas, vacation routes and reviews from experienced tourists. In this case, the web crawler technique is used to achieve this goal. As a result, the effective number of online reviews crawled for each TA is $826,841,862,808$ and 857, respectively. After preprocessing online reviews, these online reviews are split into 15,433 sentences according to the punctuation marks in reviews.

Secondly, the aspects that experienced tourists concern are extracted and the IHF information is obtained through Baidu AI platform. Since not all sentences contain opinions on TA, the opinioned 10,035 sentences in the original sentences dataset are used to construct the three-level evaluation system, as shown in Table 5 .

Meanwhile, based on the aspect-level sentiment analysis, Fig. 7 depicts the distributions of the opinioned sentences of each alternative and the percentage information of opinioned sentences with positive, neutral and negative sentiment orientations on alternative TAs concerning each sub-criterion. Figure 7a shows the percentage of positive, neutral and negative sentences in opinioned sentences for each alternative TA. For instance, the alternative $A_{1}$ contains 1530 positive sentences, 226 neutral sentences and 630 negative sentences, i.e., positive sentences account for $64.12 \%$, neutral sentences account for $9.47 \%$ and negative sentences account for $26.40 \%$. Overall, the positive sentences of the alternative $A_{1}$ have the highest proportion. In

Table 5 The three-level evaluation system

\begin{tabular}{lll}
\hline Target layer & Criteria layer & Sub-criteria layer \\
\hline The selection of TAs & Resources $\left(\mathrm{C}_{1}\right)$ & Scenery $\left(\mathrm{C}_{11}\right)$ \\
& & Climate $\left(\mathrm{C}_{12}\right)$ \\
& & Location $\left(\mathrm{C}_{13}\right)$ \\
& & Culture $\left(\mathrm{C}_{14}\right)$ \\
& Feeling $\left(\mathrm{C}_{2}\right)$ & Entertainment $\left(\mathrm{C}_{21}\right)$ \\
& & Crowd $\left(\mathrm{C}_{22}\right)$ \\
& & Emotion $\left(\mathrm{C}_{23}\right)$ \\
& & Cost $\left(\mathrm{C}_{24}\right)$ \\
& Guidance $\left(\mathrm{C}_{31}\right)$ \\
& Service $\left(\mathrm{C}_{3}\right)$ & Accommodation $\left(\mathrm{C}_{32}\right)$ \\
& & Food $\left(\mathrm{C}_{33}\right)$ \\
& Shopping $\left(\mathrm{C}_{34}\right)$ \\
& Informatization $\left(\mathrm{C}_{41}\right)$ \\
& Environment $\left(\mathrm{C}_{42}\right)$ \\
& Traffic $\left(\mathrm{C}_{43}\right)$ \\
& & Facilities $\left(\mathrm{C}_{44}\right)$ \\
&
\end{tabular}




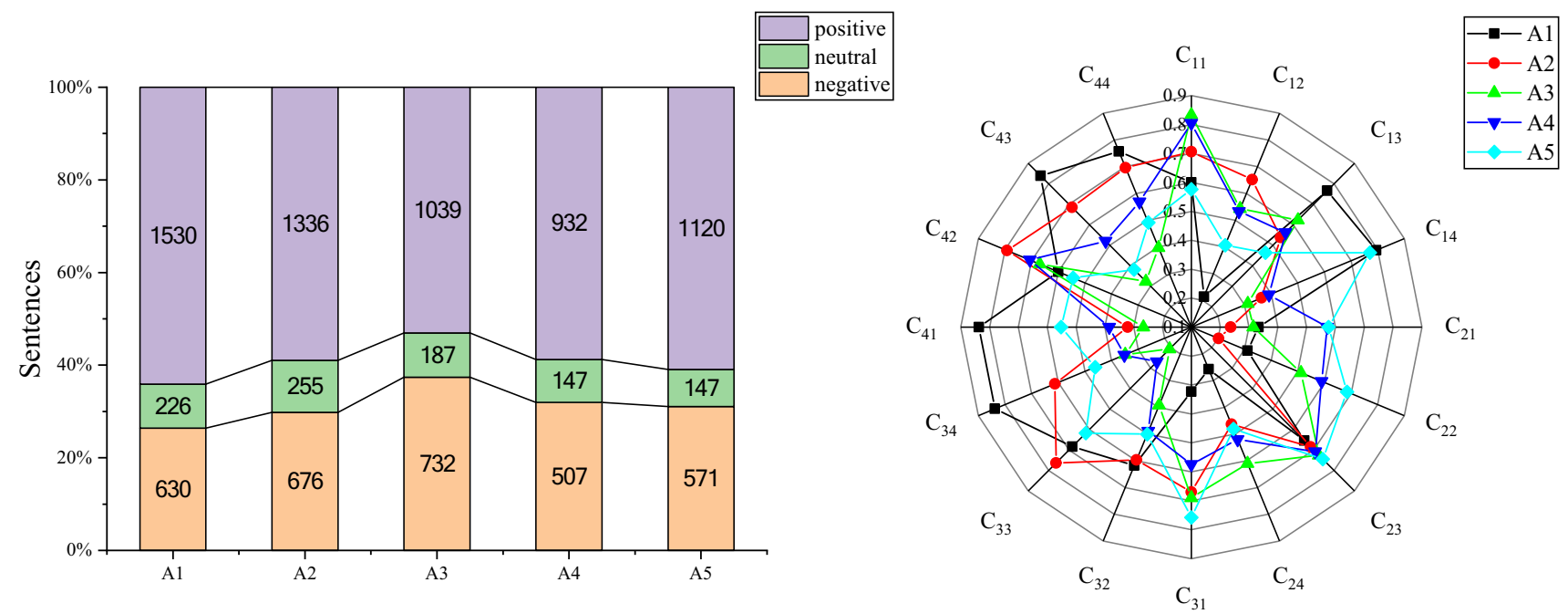

(a) Alternatives

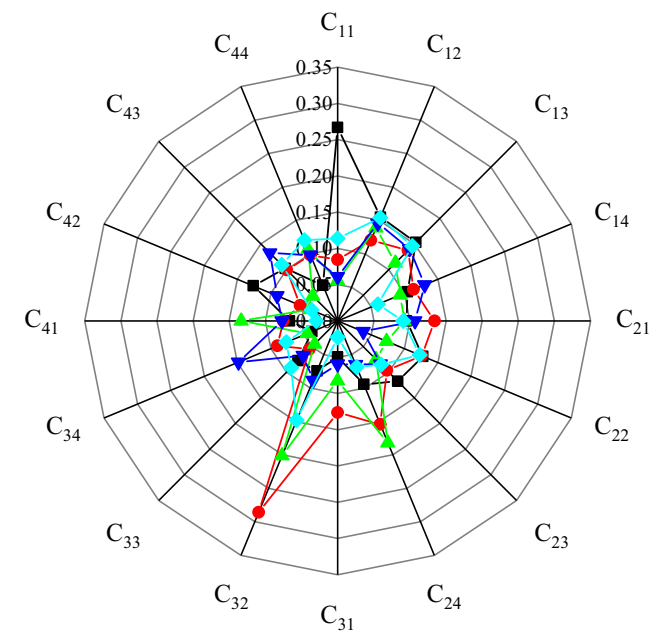

(c) Neutral

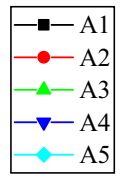

(b) Positive

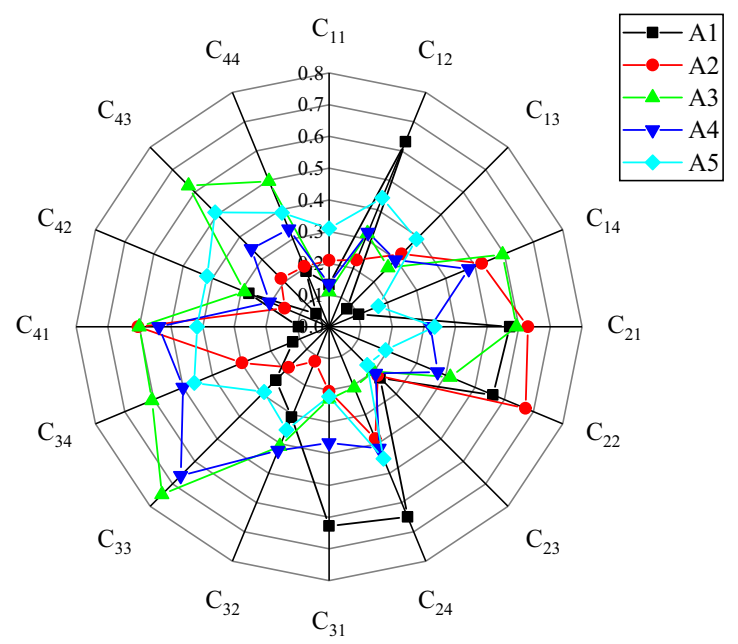

(d) Negative

Fig. 7 The distribution of opinioned sentences of each alternative and each sub-criterion

Fig. $7 b$, the experienced tourists of $A_{1}$ have the highest satisfaction with sub-criteria $\mathrm{C}_{43}$ (Traffic), and $\mathrm{A}_{2}$ is $\mathrm{C}_{42}$ (Environment), $\mathrm{A}_{3}$ and $\mathrm{A}_{4}$ are both $\mathrm{C}_{11}$ (Scenery), $\mathrm{A}_{5}$ is $\mathrm{C}_{14}$ (Culture), respectively. In Fig. 7c, the experienced tourists of $\mathrm{A}_{1}$ have the highest neutral attitude towards $\mathrm{C}_{11}$ (Scenery), and $\mathrm{A}_{2}, \mathrm{~A}_{3}$ and $\mathrm{A}_{5}$ are $\mathrm{C}_{32}$ (Accommodation), $\mathrm{A}_{4}$ is $\mathrm{C}_{34}$ (Shopping), respectively. Therefore, $\mathrm{A}_{2}, \mathrm{~A}_{3}$ and $\mathrm{A}_{5}$ should strengthen the improvement of the accommodation environment inside the TA to attract the attention of neutral tourists. According to Fig. 7d, the experienced tourists of $\mathrm{A}_{1}$ have the highest dissatisfaction with sub-criteria $\mathrm{C}_{24}$ (Cost), and $\mathrm{A}_{2}$ is $\mathrm{C}_{22}$ (Crowd), $\mathrm{A}_{3}$ is $\mathrm{C}_{33}$ (Food), $\mathrm{A}_{4}$ is $\mathrm{C}_{41}$ (Informatization), $\mathrm{A}_{5}$ is $\mathrm{C}_{43}$ (Traffic), respectively. As a result, each TA should make improvements to its weak and poor aspects. The intuitionistic fuzzy information of sub- criteria is converted to HFNs that demonstrate the performance score of each criterion of each alternative TA. Therefore, the HF-decision matrix $H=\left(h_{i j}\right)_{5 \times 4}$ is constructed as shown in Table 6.

Thirdly, the weights of criteria are determined by preference information from the potential tourist and experienced tourists. The individual preferences for criteria are given by the potential tourist according to her/his own subjective preferences. An IFPR $R=\left(r_{j j^{\prime}}\right)_{4 \times 4}$ is constructed as shown in Table 7.

The typical fractional programming method is used to calculate the weight vector of criteria from the IFPR $R=\left(r_{j j^{\prime}}\right)_{4 \times 4}$. Therefore, a nonlinear programming model is constructed as follows: 
Table 6 The HF-decision matrix $H=\left(h_{i j}\right)_{5 \times 4}$

\begin{tabular}{llllll}
\hline & $C_{1}$ & $C_{2}$ & $C_{3}$ & $C_{4}$ \\
\hline$A_{1}$ & $(0.2143,0.6000,0.7664,0.7941)$ & $(0.2568,0.3111,0.3333,0.6544)$ & $(0.3223,0.6170,0.6839,0.8373)$ & $(0.6000,0.7574,0.8367,0.8400)$ \\
$A_{2}$ & $(0.3636,0.5375,0.6519,0.7064)$ & $(0.2022,0.2371,0.4636,0.6859)$ & $(0.5963,0.6124,0.6699,0.7638)$ & $(0.3208,0.6860,0.6961,0.7920)$ \\
$A_{3}$ & $(0.3125,0.5426,0.6226,0.8333)$ & $(0.3148,0.5122,0.6104,0.7260)$ & $(0.2072,0.3484,0.3918,0.6899)$ & $(0.2667,0.3226,0.3969,0.6690)$ \\
$A_{4}$ & $(0.3913,0.5327,0.5625,0.8043)$ & $(0.5203,0.5714,0.5897,0.7079)$ & $(0.2692,0.3514,0.4889,0.5743)$ & $(0.3846,0.5204,0.5686,0.7062)$ \\
$A_{5}$ & $(0.4066,0.4634,0.5759,0.7708)$ & $(0.4809,0.5758,0.6842,0.7442)$ & $(0.4615,0.5000,0.6182,0.7575)$ & $(0.3806,0.4907,0.5437,0.5522)$ \\
\hline
\end{tabular}

$$
\begin{aligned}
& \min f=\psi_{12}+\zeta_{12}+\psi_{13}+\zeta_{13}+\psi_{14}+\zeta_{14} \\
& +\psi_{23}+\zeta_{23}+\psi_{24}+\zeta_{24}+\psi_{34}+\zeta_{34} \\
& \left\{\begin{array}{l}
\frac{\omega_{1}}{\omega_{1}+\omega_{2}}+\psi_{12}-0.35 \geq 0 \\
\frac{\omega_{1}}{\omega_{1}+\omega_{3}}+\psi_{13}-0.40 \geq 0 \\
\frac{\omega_{1}}{\omega_{1}+\omega_{4}}+\psi_{14}-0.55 \geq 0 \\
\frac{\omega_{2}}{\omega_{2}+\omega_{3}}+\psi_{23}-0.70 \geq 0
\end{array}\right. \\
& \frac{\omega_{2}}{\omega_{2}+\omega_{4}}+\psi_{24}-0.60 \geq 0 \\
& \frac{\omega_{3}}{\omega_{3}+\omega_{4}}+\psi_{34}-0.55 \geq 0 \\
& \frac{\omega_{1}}{\omega_{1}+\omega_{2}}-\zeta_{12}+0.55 \leq 1 \\
& \text { s.t. }\left\{\begin{array}{l}
\frac{\omega_{1}}{\omega_{1}+\omega_{3}}-\zeta_{13}+0.35 \leq 1 \\
\frac{\omega_{1}}{\omega_{1}+\omega_{4}}-\zeta_{14}+0.35 \leq 1
\end{array}\right. \\
& \frac{\omega_{2}}{\omega_{2}+\omega_{3}}-\zeta_{23}+0.10 \leq 1 \\
& \frac{\omega_{2}}{\omega_{2}+\omega_{4}}-\zeta_{24}+0.20 \leq 1 \\
& \frac{\omega_{3}}{\omega_{3}+\omega_{4}}-\zeta_{34}+0.30 \leq 1 \\
& \omega_{1}+\omega_{2}+\omega_{3}+\omega_{4}=1 \\
& 0 \leq \omega_{1} \leq 1,0 \leq \omega_{2} \leq 1,0 \leq \omega_{3} \leq 1,0 \leq \omega_{4} \leq 1 \\
& \psi_{12} \geq 0, \psi_{13} \geq 0, \psi_{14} \geq 0, \psi_{23} \geq 0, \psi_{24} \geq 0, \psi_{34} \geq 0 \\
& \zeta_{12} \geq 0, \zeta_{13} \geq 0, \zeta_{14} \geq 0, \zeta_{23} \geq 0, \zeta_{24} \geq 0, \zeta_{34} \geq 0 \\
& \psi_{12} \cdot \zeta_{12}=0, \psi_{13} \cdot \zeta_{13}=0, \psi_{14} \cdot \zeta_{14}=0 \\
& \psi_{23} \cdot \zeta_{23}=0, \psi_{24} \cdot \zeta_{24}=0, \psi_{34} \cdot \zeta_{34}=0
\end{aligned}
$$

Based on LINGO 17.0 software, the optimal value of the objective function is 0 , i.e., $f^{*}=0$. Therefore, the IFPR satisfies the multiplicative consistency, and the weight vector $\omega=(0.2467,0.4581,0.1623,0.1328)^{T}$ is obtained based on the preference of the potential tourist.
Table 7 The IFPR matrix $R=\left(r_{j j^{\prime}}\right)_{4 \times 4}$

\begin{tabular}{lllll}
\hline & $C_{1}$ & $C_{2}$ & $C_{3}$ & $C_{4}$ \\
\hline$C_{1}$ & $(0.50,0.50)$ & $(0.35,0.55)$ & $(0.40,0.35)$ & $(0.55,0.35)$ \\
$C_{2}$ & $(0.55,0.35)$ & $(0.50,0.50)$ & $(0.70,0.10)$ & $(0.60,0.20)$ \\
$C_{3}$ & $(0.35,0.40)$ & $(0.10,0.70)$ & $(0.50,0.50)$ & $(0.55,0.30)$ \\
$C_{4}$ & $(0.35,0.55)$ & $(0.20,0.60)$ & $(0.30,0.55)$ & $(0.50,0.50)$ \\
\hline
\end{tabular}

Table 8 The HF-entropy matrix

\begin{tabular}{lllll}
\hline & $C_{1}$ & $C_{2}$ & $C_{3}$ & $C_{4}$ \\
\hline$A_{1}$ & 0.4023 & 0.5022 & 0.4052 & 0.2953 \\
$A_{2}$ & 0.4525 & 0.4992 & 0.3919 & 0.3955 \\
$A_{3}$ & 0.4319 & 0.4593 & 0.4923 & 0.5026 \\
$A_{4}$ & 0.4442 & 0.4394 & 0.5136 & 0.4649 \\
$A_{5}$ & 0.4496 & 0.4154 & 0.4430 & 0.4995 \\
\hline
\end{tabular}

The collective preferences from experienced tourists for criteria are calculated based on the HF-entropy weight method. The HF-entropy measure of each HFN $h_{i j}$ is calculated by Eq. (8), as shown in Table 8.

The priority weight vector of criteria based on collective preferences from experienced tourists is obtained by Eq. (9), i.e., $\varpi=(0.2540,0.2418,0.2481,0.2560)^{T}$.

During the process of determining the optimal combination weight vector of criteria, the subjective weights, objective weights and final integrated weights are shown in Table 9.

Finally, the IHF-TOPSIS method is applied to rank the alternative TAs. The IF information for sub-criteria has been acquired as shown in Fig. 7, the HF-decision matrix $H=\left(h_{i j}\right)_{5 \times 4}$ has been constructed as shown in Table 6 , and the final optimal combination weight vector of criteria is calculated as shown in Table 9. After each HFN is calculated by Eq. (21), the weighted HF-decision matrix $\bar{H}=$ $\left(\bar{h}_{i j}\right)_{5 \times 4}$ is constructed as shown in Table 10. 
Table 9 The integrated weights of criteria

\begin{tabular}{lllll}
\hline Subjective weights & Objective weights & $W^{l}$ & $W^{2}$ & Integrated weights \\
\hline 0.2467 & 0.2540 & 0.2530 & 0.2503 & 0.2335 \\
0.4581 & 0.2418 & 0.4472 & 0.3500 & 0.3668 \\
0.1623 & 0.2481 & 0.1626 & 0.2052 & 0.2249 \\
0.1328 & 0.2560 & 0.1373 & 0.1944 & 0.1749 \\
\hline
\end{tabular}

Table 10 The weighted HF-decision matrix $\bar{H}=\left(\bar{h}_{i j}\right)_{5 \times 4}$

\begin{tabular}{llllll}
\hline & $C_{1}$ & $C_{2}$ & $C_{3}$ & $C_{4}$ \\
\hline$A_{1}$ & $(0.0548,0.1926,0.2879,0.3086)$ & $(0.1031,0.1278,0.1382,0.3228)$ & $(0.0838,0.1941,0.2282,0.3353)$ & $(0.1481,0.2194,0.2717,0.2742)$ \\
$A_{2}$ & $(0.1002,0.1648,0.2184,0.2489)$ & $(0.0795,0.0945,0.2043,0.3461)$ & $(0.1845,0.1920,0.2206,0.2772)$ & $(0.0654,0.1834,0.1880,0.2401)$ \\
$A_{3}$ & $(0.0838,0.1669,0.2035,0.3419)$ & $(0.1295,0.2315,0.2923,0.3781)$ & $(0.0509,0.0918,0.1058,0.2315)$ & $(0.0528,0.0658,0.0847,0.1758)$ \\
$A_{4}$ & $(0.1095,0.1627,0.1755,0.3168)$ & $(0.2362,0.2671,0.2788,0.3632)$ & $(0.0681,0.0928,0.1401,0.1747)$ & $(0.0814,0.1206,0.1368,0.1928)$ \\
$A_{5}$ & $(0.1147,0.1353,0.1815,0.2910)$ & $(0.2138,0.2699,0.3448,0.3935)$ & $(0.1300,0.1443,0.1947,0.2728)$ & $(0.0804,0.1113,0.1282,0.1311)$ \\
\hline
\end{tabular}

Table 11 The ranking results of TAs

\begin{tabular}{lll}
\hline Methods & Number of criteria & Ranking \\
\hline The proposed method & 4 & $A_{1} \succ A_{5} \succ A_{2} \succ A_{4} \succ A_{3}$ \\
Situation 1 & 4 & $A_{1} \succ A_{5} \succ A_{4} \succ A_{2} \succ A_{3}$ \\
Situation 2 & 16 & $A_{1} \succ A_{4} \succ A_{5} \succ A_{2} \succ A_{3}$ \\
\hline
\end{tabular}

The HFPI and HFNI solutions are calculated by Eqs. (22)-(23), i.e.,

$A^{+}=\left\{\begin{array}{c}\left\langle C_{1},\{0.1147,0.1926,0.2879,0.3419\}\right\rangle,\left\langle C_{2},\{0.2362,0.2699,0.3448,0.3935\}\right\rangle, \\ \left\langle C_{3},\{0.1845,0.1941,0.2282,0.3353\}\right\rangle,\left\langle C_{4},\{0.1481,0.2194,0.2717,0.2742\}\right\rangle\end{array}\right\}$

$A^{-}=\left\{\begin{array}{c}\left\langle C_{1},\{0.0548,0.1353,0.1755,0.2489\}\right\rangle,\left\langle C_{2},\{0.0795,0.0945,0.1382,0.3228\}\right\rangle, \\ \left\langle C_{3},\{0.0509,0.0918,0.1058,0.1747\}\right\rangle,\left\langle C_{4},\{0.0528,0.0658,0.0847,0.1311\}\right\rangle\end{array}\right\}$

The distance between the alternative and the HFPI or HFNI solution is calculated by Eqs. (24)-(25), i.e.,

$$
\begin{aligned}
D_{1}^{+} & =0.2310, D_{2}^{+}=0.2923, D_{3}^{+}=0.3650, D_{4}^{+} \\
& =0.3144, D_{5}^{+}=0.2477 \\
D_{1}^{-} & =0.3530, D_{2}^{-}=0.2787, D_{3}^{-}=0.2135, D_{4}^{-} \\
& =0.2534, D_{5}^{-}=0.3079
\end{aligned}
$$

Based on the HFPI solution, the relative closeness degree $C_{i}$ is calculated by Eq. (26), i.e., $C\left(A_{1}\right)=0.6045$, $C\left(A_{2}\right)=0.4881, \quad C\left(A_{3}\right)=0.3691, \quad C\left(A_{4}\right)=0.4463$, $C\left(A_{5}\right)=0.5542$. According to the obtained $C_{i}$, the ranking list is determined as $A_{1} \succ A_{5} \succ A_{2} \succ A_{4} \succ A_{3}$. Consequently, the most suitable TA for the potential tourist $A_{1}$ (i.e., the Palace Museum) is selected by the RS.

\subsection{Comparative Analysis}

In order to further validate the effectiveness of the proposed methodology, a comparative analysis is conducted with the IFS theory [56]. Situation 1: since the envelopment of HFE is an IFN, the HF decision information in Table 6 is converted into IF decision information. Situation 2: the 16 criteria are directly used to construct an IF decision matrix. As a result, the ranking results from three different methods are shown in Table 11.

Obviously, the three ranking results are different. The difference is mainly among $A_{5}, A_{2}$ and $A_{4}$. The main reasons for this result are as follows: (1) The ranking method proposed in this paper is based on IHF information and it contains several membership degrees, which not only reflects the performance score of each criterion but also well reflects the hesitation information of experienced tourists. However, in Situation 1, the HFE is transformed into the IFN, which easily loses a large amount of original decision information and ultimately affects the ranking result. (2) Although the original decision information is not lost in Situation 2, the number of criteria is larger, which makes the decision-making process more complicated and also cannot reflect the hesitation information of experienced tourists. Therefore, it also leads to the difference in the final ranking result.

In addition to comparing the different scenarios as mentioned above, we ulteriorly perform a comparative analysis with another two MCDM methods to explore the consistency of the developed approach in this paper. As aforementioned, a similar solution for the decision-making 
Table 12 Ranking results of TAs

\begin{tabular}{ll}
\hline Methods & Ranking \\
\hline The proposed method & $A_{1} \succ A_{5} \succ A_{2} \succ A_{4} \succ A_{3}$ \\
IHF-VIKOR method [56] & $A_{5} \succ A_{1} \succ A_{2} \succ A_{4} \succ A_{3}$ \\
IHF-TODIM method [58] & $A_{1} \succ A_{2} \succ A_{5} \succ A_{4} \succ A_{3}$ \\
\hline
\end{tabular}

problem was proposed by Cali and Balaman [56] and Zhang et al. [58]. In the method of [56], a novel decision support system based on the IFS theory and aspect-level sentiment analysis technique was put forward. In their research, the entropy method was employed to determine the weights of criteria objectively. Moreover, to the best of our knowledge, Zhang et al. [58] firstly combined HFS theory and sentiment analysis to rank products with online reviews. Building on the extended TODIM method, the optimal product was selected. To better demonstrate the advantages of our developed method, the existing methods of [56] and [58], as well as the proposed method in this paper, are used to rank alternative TAs, respectively. To save space, the detailed calculation process is shown in Appendix and the final ranking results through different methods are reported in Table 12.

As depicted in Table 12, the three ranking results are not consistent. The best alternative TA derived from the proposed method and the IHF-TODIM method [58] is the same, i.e., $A_{1}$. However, the ranking position of the alternative $A_{1}$ goes back and $A_{5}$ goes forward, the ranking result from the IHF-VIKOR method [56] claims that $A_{5}$ is the optimal alternative. What is more, in contrast to the proposed method in this paper, the ranking orders of the remaining alternatives by the two existing methods also have changed significantly. On the one hand, these distinctions lie in the different central ideas employed by these three MCDM methods. On the other hand, the main reason for this result is that personal preferences are not taken into consideration in the decision-making process. No matter it is the method of [56] or the method from [58], they only consider the collective preferences from experienced consumers on criteria. However, this is not in line with real life. The potential tourists usually have their own preferences for certain aspects (i.e., criteria). This personal preference information tends to have a significant impact on the final ranking results. To this end, this paper fuses the preference information from experienced tourists and the potential tourist to ensure the reliability of the final ranking. At the same time, we not only consider the intuitionistic fuzzy information in online reviews, but also consider the hesitant fuzzy information, which contributes to describe the fuzzy and uncertain information in reviews more comprehensively. Taken together, it is apparent that the proposed method in this paper is more credible as well as is superior to the existing methods.

\section{Conclusions}

In the face of a large number of online reviews from experienced tourists, how quickly and effectively assist potential tourists to make travel decisions is a very practical issue. For this purpose, this paper proposes a RS based on previous work, which combines the aspect-level sentiment analysis, IFS theory and HFS theory, aiming to rank alternative TAs through online reviews and select the suitable TA for potential tourists. The proposed methodology consists of four phases: The first phase is to crawl online reviews of alternative TAs from OTWs by using web crawler software. Secondly, the aspects that experienced tourists concern are extracted to construct a threelevel evaluation system, and we utilize aspect-level sentiment analysis to identify the IHF information of each subcriterion, where a HF-decision matrix is constructed related to each criterion. Thirdly, the preference information from the potential tourist and experienced tourists is integrated to determine the weights of criteria. The last phase, the IHF-TOPSIS, a MCDM method, is proposed to rank alternative TAs. The main innovations of this paper are concluded as follows:

First, this paper provides a new approach to objectively characterize the IHF information in online reviews based on the aspect-level sentiment analysis and proposes the IHF-TOPSIS method. Secondly, most of the existing studies use several criteria when evaluating the quality of alternatives, usually 4-6. This rough evaluation system often leads to insufficient consideration of alternatives and greatly increases the risk of decision-making. Meanwhile, the potential tourist tends to leave out consideration on a certain aspect due to the limited knowledge and expertise. Therefore, this paper extracts the aspects that experienced tourists concern from massive online reviews and constructs a three-level evaluation system, which effectively avoids the above problems. Third, this paper points out that the weights of criteria should be determined together by the potential tourist and experienced tourists. By taking the preferences of potential tourists into consideration, this paper combines the preference information from the potential tourist and experienced tourists to determine the optimal combination weight of criteria, which makes the final ranking result more reasonable. Fourth, this paper transforms massive online reviews into IHF information. Therefore, it provides a new idea for the transformation and fusion of qualitative online reviews into quantitative numerical information. 
It is worth noting that the methodology proposed in this paper is not only limited to the ranking of TAs, it has strong portability and can be applied to other situations with similar processes, such as online shopping, online booking, etc. Through conducting a comparative analysis with former methods, the result indicates that our developed method has strong practicality and obvious advantages. However, this study also exists some limitations that guide us towards the directions for future research. For example, in the construction of the HF-decision matrix, although more decisionmaking information is included to fully reflect the hesitation of experienced tourists, the non-membership degree information is not considered, which may lead to lose some decision-making information. Therefore, the non-membership degree information of each sub-criterion needs to be considered in future research. The RS proposed in this paper serves a single potential tourist but without considering the group travel. In real life, there are situations where a family or several friends travel together. Under this situation, the proposed RS is no longer applicable. Therefore, how to effectively deal with the decision-making contradictions of the group is another important research direction in the future. Moreover, along with the gradual diversification of information types and information structures expressed by tourists, how to more accurately characterize the ambiguity and complex uncertainty therein is still an important and meaningful topic in future research.

Acknowledgements The work was supported by the National Natural Science Foundation of China (Nos. 71571123 and 71771155) and the Fundamental Research Funds for the Central Universities (YJ202063, SXYPY202146).

\section{Appendix}

(1) The detailed steps of the IHF-VIKOR method are shown as below:

Step 1. Obtain the intuitionistic and hesitant fuzzy evaluation information. In order to be comparable, we use the same evaluation information in Table 6 .

Step 2. Determine the positive ideal solution (PIS) $A^{*}$ and the negative ideal solution (NIS) $A^{-}$:

$$
\begin{aligned}
& A^{*}=\left\{\left\langle h_{j}^{*}, \max _{i}\left\{h_{i j}^{\sigma(q)}\right\}\right\rangle \mid(j=1,2, \cdots, m)\right\} \\
& A^{-}=\left\{\left\langle h_{j}^{-}, \min _{i}\left\{h_{i j}^{\sigma(q)}\right\}\right\rangle \mid(j=1,2, \cdots, m)\right\}
\end{aligned}
$$

where $\quad A_{m}^{*}=\left\{\left\langle h_{m}^{*},\left\{\left(h_{m}^{1}\right)^{*},\left(h_{m}^{2}\right)^{*}, \cdots,\left(h_{m}^{l}\right)^{*}\right\}\right\rangle\right\} \quad$ and $A_{m}^{-}=\left\{\left\langle h_{m}^{-},\left\{\left(h_{m}^{1}\right)^{-},\left(h_{m}^{2}\right)^{-}, \cdots,\left(h_{m}^{l}\right)^{-}\right\}\right\rangle\right\}$.

Therefore, the $A^{*}$ and $A^{-}$will be:
$A^{*}=\left\{\begin{array}{c}\left\langle h_{1}^{*},\{0.4066,0.6000,0.7664,0.8333\}\right\rangle,\left\langle h_{2}^{*},\{0.5203,0.5758,0.6842,0.7442\}\right\rangle, \\ \left\langle h_{3}^{*},\{0.5963,0.6170,0.6839,0.8373\}\right\rangle,\left\langle h_{4}^{*},\{0.6000,0.7574,0.8367,0.8400\}\right\rangle\end{array}\right\}$

$A^{-}=\left\{\begin{array}{c}\left\langle h_{1}^{-},\{0.2143,0.4634,0.5625,0.7064\}\right\rangle,\left\langle h_{2}^{-},\{0.2022,0.2371,0.3333,0.6544\}\right\rangle, \\ \left\langle h_{3}^{-},\{0.2072,0.3484,0.3918,0.5743\}\right\rangle,\left\langle h_{4}^{-},\{0.2667,0.3226,0.3969,0.5522\}\right\rangle\end{array}\right\}$

Step 3. Determine the weights of criteria. In the method of [56], the entropy measure was employed to calculate the weights of criteria. Therefore, the criteria weight is $w=(0.2540,0.2418,0.2481,0.2560)$.

Step 4. Compute the $S_{i}$ and $R_{i}$ indexes values for alternatives.

$S_{i}=\sum_{j=1}^{m} w_{j} \frac{d\left(h_{j}^{*}, h_{i j}\right)}{d\left(h_{j}^{*}, h_{j}^{-}\right)}, i=1,2, \cdots, n$

$R_{i}=\max _{j}\left(w_{j} \frac{d\left(h_{j}^{*}, h_{i j}\right)}{d\left(h_{j}^{*}, h_{j}^{-}\right)}\right), i=1,2, \cdots, n$

Here, we use the Euclidean distance to calculate distances between different HFEs. As a result, the $S_{i}$ index values are obtained as follows:

$$
\begin{aligned}
S_{1}= & w_{1} \frac{d\left(h_{1}^{*}, h_{11}\right)}{d\left(h_{1}^{*}, h_{1}^{-}\right)}+w_{2} \frac{d\left(h_{2}^{*}, h_{12}\right)}{d\left(h_{2}^{*}, h_{2}^{-}\right)}+w_{3} \frac{d\left(h_{3}^{*}, h_{13}\right)}{d\left(h_{3}^{*}, h_{3}^{-}\right)} \\
& +w_{4} \frac{d\left(h_{4}^{*}, h_{14}\right)}{d\left(h_{4}^{*}, h_{4}^{-}\right)} \\
= & 0.4722
\end{aligned}
$$

$S_{2}=0.4932, S_{3}=0.7062, S_{4}=0.5687, S_{5}=0.4634$

Besides, the $R_{i}$ index values are formulated as follows: $R_{1}=\max _{4}\left\{w_{1} \frac{d\left(h_{1}^{*}, h_{11}\right)}{d\left(h_{1}^{*}, h_{1}^{-}\right)}, w_{2} \frac{d\left(h_{2}^{*}, h_{12}\right)}{d\left(h_{2}^{*}, h_{2}^{-}\right)}, w_{3} \frac{d\left(h_{3}^{*}, h_{13}\right)}{d\left(h_{3}^{*}, h_{3}^{-}\right)}, w_{4} \frac{d\left(h_{4}^{*}, h_{14}\right)}{d\left(h_{4}^{*}, h_{4}^{-}\right)}\right\}=0.2135$

$R_{2}=0.2125, R_{3}=0.2438, R_{4}=0.2153, R_{5}=0.1830$

Accordingly, the $S^{*}, S^{-}, R^{*}$ and $R^{-}$indexes will be:

$S^{*}=\max S_{i}=0.7062, \quad S^{-}=\min S_{i}=0.4634$

$R^{*}=\max R_{i}=0.2438, \quad R^{-}=\min R_{i}=0.1830$

Step 5 Compute the $Q_{i}$ values by Eq. (31):

$\begin{aligned} Q_{i}= & v\left(S_{i}-S^{-}\right) /\left(S^{*}-S^{-}\right)+(1 \\ & -v)\left(R_{i}-R^{-}\right) /\left(R^{*}-R^{-}\right)\end{aligned}$

where $v$ depicts the strategic weight. Let $v=0.5$, the $Q_{i}(i=1,2,3,4,5)$ values for five alternatives are as follows:

$Q_{1}=0.2691, \quad Q_{2}=0.3034, \quad Q_{3}=1, \quad Q_{4}=0.4824$,

$Q_{5}=0$

Step 6 Rank the alternatives. In this step, the optimal alternative is considered as the alternative with the lowest values of $S, R$ and $Q$. According to values of $S, R$ and $Q$, the ranking results are given in Table 13 . 
Table 13 The ranking results for alternatives

\begin{tabular}{|c|c|c|c|c|c|c|}
\hline & $A_{1}$ & $A_{2}$ & $A_{3}$ & $A_{4}$ & $A_{5}$ & Ranking \\
\hline$S$ & 0.4722 & 0.4932 & 0.7062 & 0.5687 & 0.4634 & $A_{5} \succ A_{1} \succ A_{2} \succ A_{4} \succ A_{3}$ \\
\hline$R$ & 0.2135 & 0.2125 & 0.2438 & 0.2153 & 0.1830 & $A_{5} \succ A_{2} \succ A_{1} \succ A_{4} \succ A_{3}$ \\
\hline$Q(v=0.5)$ & 0.2691 & 0.3034 & 1 & 0.4824 & 0 & $A_{5} \succ A_{1} \succ A_{2} \succ A_{4} \succ A_{3}$ \\
\hline
\end{tabular}

Step 7 Determine the desirable solution. The final result meets the following two conditions simultaneously:

c1: Acceptable advantage: $Q\left(A^{2}\right)-Q\left(A^{1}\right) \geq \frac{1}{n-1}$, where $A^{1}$ and $A^{2}$ are the alternatives with the first and second positions in the ranking outcome; $n$ is the number of alternatives.

c2: Acceptable stability in decision-making. The alternative $A^{1}$ is the best ranked by $S$ and/or $R$.

Thus, the ranking of the five alternative TAs could be determined by $Q$, i.e., $A_{5} \succ A_{1} \succ A_{2} \succ A_{4} \succ A_{3}$.

(2) The detailed steps of the IHF-TODIM method are shown as below:

Step 1 Acquire the intuitionistic and hesitant fuzzy evaluation information described in Table 6 .

Step 2 Determine the relative weight by Eq. (32):

$\tilde{w}_{j}=\frac{w_{j}}{\hat{w}} \quad j=1, \cdots, m$

where $\hat{w}$ denotes the reference weight and $\hat{w}=\max \left(w_{j} \mid j=1, \cdots, m\right)$. According to the initial weight $w=(0.2540,0.2418,0.2481,0.2560)$, the relative criteria weights are calculated as follows:

$\tilde{w}_{1}=\frac{w_{1}}{\hat{w}}=\frac{0.2540}{0.2560}=0.9922$

$\tilde{w}_{2}=0.9445, \tilde{w}_{3}=0.9691, \tilde{w}_{4}=1$

Step 3 Compute the preference index value of the alternative $A_{i}$ over the alternative $A_{i^{\prime}}$ under criterion $C_{j}$ by Eq. (33):

$$
\phi_{j}\left(A_{i}, A_{i^{\prime}}\right)=\left\{\begin{array}{c}
-\frac{1}{\chi} \sqrt{\frac{\left(\sum_{j=1}^{m} \tilde{w}_{j}\right) d\left(h_{i j}-h_{i^{\prime} j}\right)}{\tilde{w}_{j}}} \text { if } h_{i j}<h_{i^{\prime} j} \\
0 \text { if } h_{i j}=h_{i^{\prime} j} \\
\sqrt{\frac{\tilde{w}_{j} d\left(h_{i j}-h_{i^{\prime} j}\right)}{\sum_{j=1}^{m} \tilde{w}_{j}}} \text { if } h_{i j}>h_{i^{\prime} j}
\end{array}\right.
$$

where the Euclidean distance measure on HFEs is adopted, as shown in Eq. (20), and $\chi$ represents the attenuation factor of the losses. In this paper, we take $\chi=1$.

At the same time, we compare the HFEs by the score function, i.e.,
Table 14 Preference index values for the first alternative

\begin{tabular}{lllll}
\hline & $\phi_{1}\left(A_{i}, A_{i^{\prime}}\right)$ & $\phi_{2}\left(A_{i}, A_{i^{\prime}}\right)$ & $\phi_{3}\left(A_{i}, A_{i^{\prime}}\right)$ & $\phi_{4}\left(A_{i}, A_{i^{\prime}}\right)$ \\
\hline$\left(A_{1}, A_{2}\right)$ & 0.1660 & -0.5797 & -0.7565 & 0.2038 \\
$\left(A_{1}, A_{3}\right)$ & 0.1543 & -0.8562 & 0.2333 & 0.3042 \\
$\left(A_{1}, A_{4}\right)$ & 0.1881 & -0.9685 & 0.2296 & 0.2369 \\
$\left(A_{1}, A_{5}\right)$ & 0.1965 & -1.0182 & 0.1611 & 0.2621 \\
\hline
\end{tabular}

Table 15 Preference index values for the second alternative

\begin{tabular}{rrrlr}
\hline & $\phi_{1}\left(A_{i}, A_{i^{\prime}}\right)$ & $\phi_{2}\left(A_{i}, A_{i^{\prime}}\right)$ & \multicolumn{1}{c}{$\phi_{3}\left(A_{i}, A_{i^{\prime}}\right)$} & $\phi_{4}\left(A_{i}, A_{i^{\prime}}\right)$ \\
\hline$\left(A_{2}, A_{1}\right)$ & -0.6533 & 0.1402 & 0.1877 & -0.7958 \\
$\left(A_{2}, A_{3}\right)$ & -0.5250 & -0.8309 & 0.2615 & 0.2503 \\
$\left(A_{2}, A_{4}\right)$ & -0.5165 & -0.9951 & 0.2475 & 0.1733 \\
$\left(A_{2}, A_{5}\right)$ & 0.1292 & -1.0110 & 0.1507 & 0.2116
\end{tabular}

Table 16 Preference index values for the third alternative

\begin{tabular}{rrrrr}
\hline & $\phi_{1}\left(A_{i}, A_{i^{\prime}}\right)$ & $\phi_{2}\left(A_{i}, A_{i^{\prime}}\right)$ & $\phi_{3}\left(A_{i}, A_{i^{\prime}}\right)$ & $\phi_{4}\left(A_{i}, A_{i^{\prime}}\right)$ \\
\hline$\left(A_{3}, A_{1}\right)$ & -0.6075 & 0.2070 & -0.9403 & -1.1883 \\
$\left(A_{3}, A_{2}\right)$ & 0.1334 & 0.2009 & -1.0540 & -0.9777 \\
$\left(A_{3}, A_{4}\right)$ & 0.1148 & -0.6677 & -0.5736 & -0.7521 \\
$\left(A_{3}, A_{5}\right)$ & 0.1360 & -0.6324 & -0.8737 & -0.7348 \\
\hline
\end{tabular}

$s\left(h_{i j}\right)=\frac{1}{h_{i j}} \sum_{t=1}^{h_{i j}} h_{i j}^{t}$

where $\# h_{i j}$ denotes the number of the elements in $h_{i j}$ (Tables 14, 15, 16,17, 18).

Hence, the preference index value $\phi_{j}\left(A_{i}, A_{i^{\prime}}\right)$ is calculated as follows:

Step 4 Work out the dominance degree of the alternative $A_{i}$ over the alternative $A_{i^{\prime}}$ according to Eq. (35):

$\theta_{i}=\theta\left(A_{i}, A_{i^{\prime}}\right)=\sum_{j=1}^{m} \phi_{j}\left(A_{i}, A_{i^{\prime}}\right), \quad i, i^{\prime} \in\{1, \cdots, n\}$

Thus, the dominance degrees of five alternative TAs are obtained as follows:

$\begin{aligned} \theta_{1} & =-1.8433, \theta_{2}=-3.5757, \theta_{3}=-8.2100, \theta_{4} \\ & =-4.6625, \theta_{5}=-4.5114\end{aligned}$ 
Table 17 Preference index values for the fourth alternative

\begin{tabular}{rrrrr}
\hline & $\phi_{1}\left(A_{i}, A_{i^{\prime}}\right)$ & $\phi_{2}\left(A_{i}, A_{i^{\prime}}\right)$ & $\phi_{3}\left(A_{i}, A_{i^{\prime}}\right)$ & $\phi_{4}\left(A_{i}, A_{i^{\prime}}\right)$ \\
\hline$\left(A_{4}, A_{1}\right)$ & -0.7404 & 0.2342 & -0.9255 & -0.9255 \\
$\left(A_{4}, A_{2}\right)$ & 0.1312 & 0.2406 & -0.9975 & -0.6770 \\
$\left(A_{4}, A_{3}\right)$ & -0.4519 & 0.1615 & 0.1423 & 0.1926 \\
$\left(A_{4}, A_{5}\right)$ & 0.1006 & -0.4741 & -0.8163 & 0.1426 \\
\hline
\end{tabular}

Table 18 Preference index values for the fifth alternative

\begin{tabular}{lrlrr}
\hline & $\phi_{1}\left(A_{i}, A_{i^{\prime}}\right)$ & $\phi_{2}\left(A_{i}, A_{i^{\prime}}\right)$ & $\phi_{3}\left(A_{i}, A_{i^{\prime}}\right)$ & $\phi_{4}\left(A_{i}, A_{i^{\prime}}\right)$ \\
\hline$\left(A_{5}, A_{1}\right)$ & -0.7736 & 0.2462 & -0.6493 & -1.0237 \\
$\left(A_{5}, A_{2}\right)$ & -0.5084 & 0.2445 & -0.6073 & -0.8266 \\
$\left(A_{5}, A_{3}\right)$ & -0.5355 & 0.1529 & 0.2168 & 0.1881 \\
$\left(A_{5}, A_{4}\right)$ & -0.3958 & 0.1146 & 0.2026 & -0.5569 \\
\hline
\end{tabular}

Step 5 Calculate the overall dominance for each alternative TA by Eq. (36):

$\psi_{i}=\frac{\theta_{i}-\min \theta_{i}}{\max \theta_{i}-\min \theta_{i}}, \quad i=1, \cdots, n$

Consequently, the overall dominance degrees for five alternative TAs are collected, i.e.,

$\psi_{1}=1, \psi_{2}=0.7279, \psi_{3}=0, \psi_{4}=0.5572, \psi_{5}=0.5809$

Step 6 Derive the final ranking list. Due to the fact that $\psi_{1}>\psi_{2}>\psi_{5}>\psi_{4}>\psi_{3}$, the final ranking is determined, i.e., $A_{1} \succ A_{2} \succ A_{5} \succ A_{4} \succ A_{3}$.

\section{References}

1. Hou, Z.P., Cui, F.S., Meng, Y.H., Lian, T.H., Yu, C.H.: Opinion mining from online travel reviews: A comparative analysis of Chinese major OTAs using semantic association analysis. Tour. Manage. 74, 276-289 (2019)

2. Li, J.J., Xu, L.Z., Tang, L., Wang, S.Y., Li, L.: Big data in tourism research: A literature review. Tour. Manage. 68, 301-323 (2018)

3. Afzaal, M., Usman, M., Fong, A.: Predictive aspect-based sentiment classification of online tourist reviews. J. Inf. Sci. 45(3), 341-363 (2019)

4. Toral, S.L., Martinez-Torres, M.R., Gonzalez-Rodriguez, M.R.: Identification of the unique attributes of tourist destinations from online reviews. J. Travel Res. 57(7), 908-919 (2018)

5. Chong, A.Y.L., Khong, K.W., Ma, T., McCabe, S., Wang, Y.: Analyzing key influences of tourists' acceptance of online reviews in travel decisions. Internet Res. 28(3), 564-586 (2018)
6. Hu, Y.H., Chen, Y.L., Chou, H.L.: Opinion mining from online hotel reviews-A text summarization approach. Inf. Process. Manage. 53(2), 436-449 (2017)

7. Li, G., Law, R., Vu, H.Q., Rong, J.: Discovering the hotel selection preferences of Hong Kong inbound travelers using the Choquet Integral. Tour. Manage. 36, 321-330 (2013)

8. Liu, Y., Bi, J.W., Fan, Z.P.: Ranking products through online reviews: A method based on sentiment analysis technique and intuitionistic fuzzy set theory. Information Fusion 36, 149-161 (2017)

9. Zhang, D., Li, Y., Wu, C.: An extended TODIM method to rank products with online reviews under intuitionistic fuzzy environment. J. Operat. Res. Soc. (2019). https://doi.org/10.1080/ 01605682.2018.1545519

10. Valdivia, A., Hrabova, E., Chaturvedi, I., Luzon, M.V., Troiano, L., Cambria, E., Herrera, F.: Inconsistencies on TripAdvisor reviews: A unified index between users and sentiment analysis methods. Neurocomputing 353, 3-16 (2019)

11. Tripathy, A., Anand, A., Rath, S.K.: Document-level sentiment classification using hybrid machine learning approach. Knowl. Inf. Syst. 53(3), 805-831 (2017)

12. Moraes, R., Valiati, J.F., Neto, W.P.G.: Document-level sentiment classification: An empirical comparison between SVM and ANN. Expert Syst. Appl. 40(2), 621-633 (2013)

13. Xia, R., Zong, C.Q., Li, S.S.: Ensemble of feature sets and classification algorithms for sentiment classification. Inf. Sci. 181(6), 1138-1152 (2011)

14. Liu, S.M., Chen, J.H.: A multi-label classification based approach for sentiment classification. Expert Syst. Appl. 42(3), 1083-1093 (2015)

15. Onan, A., Korukoglu, S., Bulut, H.: A multiobjective weighted voting ensemble classifier based on differential evolution algorithm for text sentiment classification. Expert Syst. Appl. 62, 1-16 (2016)

16. Liu, Y., Bi, J.W., Fan, Z.P.: A method for multi-class sentiment classification based on an improved one-vs-one (OVO) strategy and the support vector machine (SVM) algorithm. Inf. Sci. 394, 38-52 (2017)

17. Abdi, A., Shamsuddin, S.M., Hasan, S., Piran, J.: Deep learningbased sentiment classification of evaluative text based on multifeature fusion. Inf. Process. Manage. 56(4), 1245-1259 (2019)

18. Do, H.H., Prasad, P.W.C., Maag, A., Alsadoon, A.: Deep learning for aspect-based sentiment analysis: a comparative review. Expert Syst. Appl. 118, 272-299 (2019)

19. Khan, F.H., Qamar, U., Bashir, S.: eSAP: A decision support framework for enhanced sentiment analysis and polarity classification. Inf. Sci. 367, 862-873 (2016)

20. Ain, Q.T., Ali, M., Riaz, A., Noureen, A., Kamran, M., Hayat, B., Rehman, A.: Sentiment analysis using deep learning techniques: A review. Int. J. Adv. Comput. Sci. Appl. 8(6), 424-433 (2017)

21. Zadeh, L.A.: Fuzzy sets. Inf. Control 8(3), 338-353 (1965)

22. Turksen, I.B.: Interval valued fuzzy-sets based on normal forms. Fuzzy Sets Syst. 20(2), 191-210 (1986)

23. Atanassov, K.T.: Intuitionistic fuzzy sets. Fuzzy Sets Syst. 20(1), 87-96 (1986)

24. Torra, V.: Hesitant fuzzy sets. Int. J. Intell. Syst. 25(6), 529-539 (2010)

25. Yager, R.R.: Pythagorean membership grades in multicriteria decision making. IEEE Trans. Fuzzy Syst. 22(4), 958-965 (2014)

26. Liu, H.W., Wang, G.J.: Multi-criteria decision-making methods based on intuitionistic fuzzy sets. Eur. J. Oper. Res. 179(1), 220-233 (2007)

27. Hu, J., Zhang, X., Yang, Y., Liu, Y., Chen, X.: New doctors ranking system based on VIKOR method. Int. Trans. Oper. Res. 27(2), 1236-1261 (2020) 
28. Mishra, A.R., Mardani, A., Rani, P., Zavadskas, E.K.: A novel EDAS approach on intuitionistic fuzzy set for assessment of health-care waste disposal technology using new parametric divergence measures. J. Cleaner Prod. 272, 122807 (2020)

29. Pei, Z., Zheng, L.: A novel approach to multi-attribute decision making based on intuitionistic fuzzy sets. Expert Syst. Appl. 39(3), 2560-2566 (2012)

30. Wu, M.C., Chen, T.Y.: The ELECTRE multicriteria analysis approach based on Atanassov's intuitionistic fuzzy sets. Expert Syst. Appl. 38(10), 12318-12327 (2011)

31. Zhang, K., Huang, Y., Yuan, X., Ma, H., Zhao, C.: Infrared and visible image fusion based on intuitionistic fuzzy sets. Infrared Phys. Technol. 105, 103124 (2020)

32. Wei, G.W.: Some similarity measures for picture fuzzy sets and their applications. Iranian Journal of Fuzzy Systems 15, 77-89 (2018)

33. Hu, K., Tan, Q., Zhang, T., Wang, S.: Assessing technology portfolios of clean energy-driven desalination-irrigation systems with interval-valued intuitionistic fuzzy sets. Renew. Sustain. Energy Rev. 132, 109950 (2020)

34. Xia, M.M., Xu, Z.S.: Hesitant fuzzy information aggregation in decision making. Int. J. Approximate Reasoning 52(3), 395-407 (2011)

35. Xu, Z.S., Zhang, S.: An overview on the applications of the hesitant fuzzy sets in group decision-making: Theory, support and methods. Front. Eng. Manag. 6(2), 163-182 (2019)

36. Liao, H.C., Xu, Z.S.: Some new hybrid weighted aggregation operators under hesitant fuzzy multi-criteria decision making environment. Journal of Intelligent \& Fuzzy Systems 26, 1601-1617 (2014)

37. Xu, Z.S., Xia, M.M.: Distance and similarity measures for hesitant fuzzy sets. Inf. Sci. 181(11), 2128-2138 (2011)

38. Xu, Z.S., Xia, M.M.: On distance and correlation measures of hesitant fuzzy information. Int. J. Intell. Syst. 26(5), 410-425 (2011)

39. Mishra, A.R., Rani, P., Pardasani, K.R., Mardani, A.: A novel hesitant fuzzy WASPAS method for assessment of green supplier problem based on exponential information measures. J. Clean. Prod. 238, 117901 (2019)

40. Liao, H.C., Xu, Z.S.: A VIKOR-based method for hesitant fuzzy multi-criteria decision making. Fuzzy Optim. Decis. Making 12(4), 373-392 (2013)

41. Mardani, A., Saraji, M.K., Mishra, A.R., Rani, P.: A novel extended approach under hesitant fuzzy sets to design a framework for assessing the key challenges of digital health interventions adoption during the COVID-19 outbreak. Appl. Soft Comput. 96, 106613 (2020)

42. Xu, Z.S.: Deviation square priority method for distinct preference structures based on generalized multiplicative consistency. IEEE Trans. Fuzzy Syst. 23(4), 1164-1180 (2015)

43. Chiclana, F., Herrera, F., Herrera-Viedma, E.: Integrating three representation models in fuzzy multipurpose decision making based on fuzzy preference relations. Fuzzy Sets Syst. 97(1), 33-48 (1998)

44. Saaty, T.L.: Axiomatic foundation of the analytic hierarchy process. Manage. Sci. 32(7), 841-855 (1986)

45. Xu, Z.S.: Intuitionistic preference relations and their application in group decision making. Inf. Sci. 177(11), 2363-2379 (2007)

46. Zhang, K., Narayanan, R., \& Choudhary, A. (2010). Voice of the customers: mining online customer reviews for product featurebased ranking. Wosn10 Usenix, 11-11.

47. Zhang, K., Yu, C., Liao, W. K., \& Choudhary, A. (2011). Mining millions of reviews: A technique to rank products based on importance of reviews. Proceedings of the 13th international conference on electronic commerce, Liverpool, United Kingdom.
48. Peng, Y., Kou, G., Li, J.: A fuzzy PROMETHEE approach for mining customer reviews in Chinese. Arab. J. Sci. Eng. 39(6), 5245-5252 (2014)

49. Abirami, A.M., Askarunisa, A.: Sentiment analysis model to emphasize the impact of online reviews in healthcare industry. Online Inf. Rev. 41(4), 471-486 (2017)

50. Chen, K., Kou, G., Shang, J., Chen, Y.: Visualizing market structure through online product reviews: Integrate topic modeling, TOPSIS, and multi-dimensional scaling approaches. Electron. Commer. Res. Appl. 14(1), 58-74 (2015)

51. Kang, D., Park, Y.: Review-based measurement of customer satisfaction in mobile service: Sentiment analysis and VIKOR approach. Expert Syst. Appl. 41(4), 1041-1050 (2014)

52. Najmi, E., Hashmi, K., Malik, Z., Rezgui, A., Khan, H.U.: CAPRA: a comprehensive approach to product ranking using customer reviews. Computing 97(8), 843-867 (2015)

53. Liu, Y., Bi, J.W., Fan, Z.P.: A method for ranking products through online reviews based on sentiment classification and interval-valued intuitionistic fuzzy TOPSIS. Int. J. Inf. Technol. Decis. Mak. 16(6), 1497-1522 (2017)

54. Bi, J.W., Liu, Y., Fan, Z.P.: Representing sentiment analysis results of online reviews using interval type-2 fuzzy numbers and its application to product ranking. Inf. Sci. 504, 293-307 (2019)

55. Yang, Z., Xiong, G., Cao, Z., Li, Y., Huang, L.: A decision method for online purchases considering dynamic information preference based on sentiment orientation classification and discrete DIFWA operators. IEEE Access 7, 77008-77026 (2019)

56. Cali, S., Balaman, S.Y.: Improved decisions for marketing, supply and purchasing: Mining big data through an integration of sentiment analysis and intuitionistic fuzzy multi criteria assessment. Comput. Ind. Eng. 129, 315-332 (2019)

57. $\mathrm{Wu}, \mathrm{C}$., Zhang, D.: Ranking products with IF-based sentiment word framework and TODIM method. Kybernetes 48(5), 990-1010 (2019)

58. Zhang, D., Wu, C., Liu, J.: Ranking products with online reviews: A novel method based on hesitant fuzzy set and sentiment word framework. J Operat Res Soc (2019). https://doi.org/10.1080/ 01605682.2018.1557021

59. Liang, R.X., Wang, J.Q.: A linguistic intuitionistic cloud decision support model with sentiment analysis for product selection in E-commerce. Int. J. Fuzzy Syst. 21(3), 963-977 (2019)

60. Luo, Y., Zhang, X., Qin, Y., Yang, Z., Liang, Y.: Tourism attraction selection with sentiment analysis of online reviews based on probabilistic linguistic term sets and the IDOCRIWCOCOSO model. Int. J. Fuzzy Syst. 23(1), 295-308 (2021)

61. Khan, A., Abosuliman, S.S., Abdullah, S., Ayaz, M.: A decision support model for hotel recommendation based on the online consumer reviews using logarithmic spherical hesitant fuzzy information. Entropy 23(4), 432 (2021)

62. Cao, Q., Liu, X.D., Wang, Z.W., Zhang, S.T., Wu, J.: Recommendation decision-making algorithm for sharing accommodation using probabilistic hesitant fuzzy sets and bipartite network projection. Complex \& Intelligent Systems 6(2), 431-445 (2020)

63. Liang, D.C., Dai, Z.Y., Wang, M.W., Li, J.J.: Web celebrity shop assessment and improvement based on online review with probabilistic linguistic term sets by using sentiment analysis and fuzzy cognitive map. Fuzzy Optim. Decis. Making 19(4), 561-586 (2020)

64. Liang, D.C., Dai, Z.Y., Wang, M.W.: Assessing customer satisfaction of $\mathrm{O} 2 \mathrm{O}$ takeaway based on online reviews by integrating fuzzy comprehensive evaluation with AHP and probabilistic linguistic term sets. Appl. Soft Comput. 98, 14 (2021)

65. Liao, H.C., Xu, Z.S.: Priorities of intuitionistic fuzzy preference relation based on multiplicative consistency. IEEE Trans. Fuzzy Syst. 22(6), 1669-1681 (2014) 
66. Liao, H., Xu, Z.: Automatic procedures for group decision making with intuitionistic fuzzy preference relations. J. Intelli. Fuzzy Sys. 27(5), 2341-2353 (2014)

67. Shannon, C.E.: A mathematical theory of communication. Bell Syst. Tech. J. 27(4), 623-656 (1948)

68. Zeleny, M.: The attribute-dynamic attitude model (Adam). Manage. Sci. 23(1), 12-26 (1976)

69. Xu, Z.S., Xia, M.M.: Hesitant fuzzy entropy and cross-entropy and their use in multiattribute decision-making. Int. J. Intell. Syst. 27(9), 799-822 (2012)

70. Alemi-Ardakani, M., Milani, A.S., Yannacopoulos, S., Shokouhi, G.: On the effect of subjective, objective and combinative weighting in multiple criteria decision making: A case study on impact optimization of composites. Expert Syst. Appl. 46, 426-438 (2016)

71. Luo, Y.Y., Qin, Y., Wang, Z., Wang, J.: A dynamical system study for the ecological development of mineral resources in mineral areas. Discrete and Continuous Dynamical SystemsSeries S 12(4-5), 1073-1089 (2019)

72. Xu, Z.S., Xia, M.M.: Induced generalized intuitionistic fuzzy operators. Knowl.-Based Syst. 24(2), 197-209 (2011)

73. Liao, H.C., Xu, Z.S.: Subtraction and division operations over hesitant fuzzy sets. J. Intelli. Fuzzy Sys. 27(1), 65-72 (2014)

74. Hwang, C.L., Yoon, K.P.: Multiple attribute decision making Methods and applications A state-of-the-art survey. Eu. J. Opera. Res. 4(4), 287-288 (1981)

75. Fu, Z.G., Liao, H.C.: Unbalanced double hierarchy linguistic term set: The TOPSIS method for multi-expert qualitative decision making involving green mine selection. Information Fusion 51, 271-286 (2019)

76. Garg, D., Sidhu, J., Rani, S.: Improved TOPSIS: A multi-criteria decision making for research productivity in cloud security. Computer Standards \& Interfaces 65, 61-78 (2019)

77. Garg, H., Kaur, G.: TOPSIS based on nonlinear-programming methodology for solving decision-making problems under cubic intuitionistic fuzzy set environment. Comput. Appl. Math. 38(3), 19 (2019)

78. Gupta, P., Mehlawat, M.K., Grover, N., Pedrycz, W.: Multi-attribute group decision making based on extended TOPSIS method under interval-valued intuitionistic fuzzy environment. Appl. Soft Comput. 69, 554-567 (2018)

79. Sun, G.D., Guan, X., Yi, X., Zhou, Z.: An innovative TOPSIS approach based on hesitant fuzzy correlation coefficient and its applications. Appl. Soft Comput. 68, 249-267 (2018)

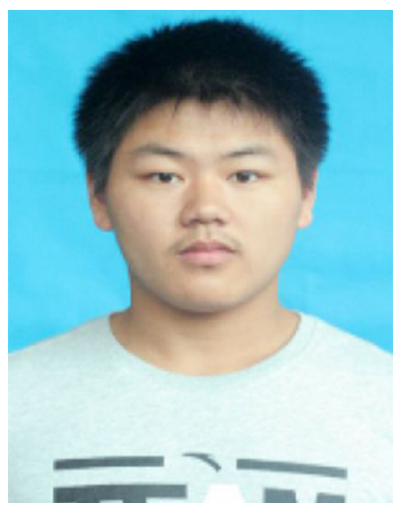

Yong Qin is currently working toward the Ph.D. degree in Business School, Sichuan University, China. His research interests include decision making, optimization, information fusion and bibliometrics. His research works have been published in International Entrepreneurship and Management Journal, Economic Research-Ekonomska Istrazivanja, Journal of Cleaner Production, International Journal of Fuzzy Systems, Symmetry, etc.

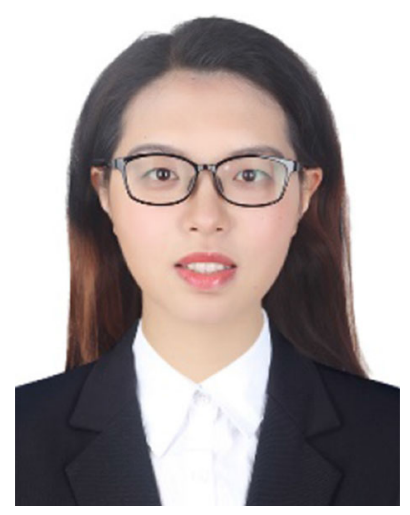

Xinxin Wang received the $\mathrm{Ph} . \mathrm{D}$. degree in Management Science and Engineering at Sichuan University, Sichuan, China. Her research interests include decision analysis, optimization, computing with words. She has published more than 20 papers, and her research works has been published in IEEE Transaction on Fuzzy Systems, Information Science sand Knowledge-Based Systems, Applied Intelligence and International Journal of Fuzzy Systems, etc. She has received numerous honors and awards, including the First Prize in National Post-Graduate Mathematical Contest in Modeling in 2015 and 2016, the 2015and 2016 National scholarship for graduate students, 2017 Presidential Scholarship at Southwest Petroleum University, etc.



Zeshui Xu received the Ph.D. degree in management science and engineering from Southeast University, Nanjing, China, in 2003. From April 2003 to May 2005, he was a Postdoctoral Researcher with the School of Economics and Management, Southeast University. From October 2005 to December 2007, he was a Postdoctoral Researcher with the School of Economics and Management, Tsinghua University, Beijing, China. He is the Distinguished Young Scholar of the National Natural Science Foundation of China and the Chang Jiang Scholars of the Ministry of Education of China. $\mathrm{He}$ is currently a Professor with the Business School, Sichuan University, Chengdu, China and also with the College of Sciences, PLA University of Science and Technology, Nanjing, China. He has been selected as a "The World's Most Influential Scientific Minds", "Thomson Reuters Highly Cited Researcher", and "Most Cited Chinese Researchers" (ranked first in Computer Science, released by Elsevier) between 2014 and 2016. He has authored nine monographs published by Springer and contributed more than 690 journal articles to professional journals, among which there are more than $460 \mathrm{SCI}$ or SSCI papers. The published papers have been cited over 48000 times, and his h-index of all published papers is 112 . He is currently the IEEE Fellow, IFSA Fellow, the Editor-in-Chief of Scholars Journal of Economics, Business and Management, and an Associate Editor or Editorial Board Member for more than 40 professional journals including IEEE Transaction on Fuzzy Systems, Information Sciences and Knowledge-Based Systems, etc. His research interests include information fusion, group decision making, computing with words, and aggregation operators. 